\title{
Representation of Infinite-Dimensional Forward Price Models in Commodity Markets
}

\author{
Fred Espen Benth • Paul Krühner
}

Received: 12 March 2014 / Revised: 28 April 2014 / Accepted: 6 May 2014 /

Published online: 26 June 2014

(C) School of Mathematical Sciences, University of Science and Technology of China and Springer-Verlag Berlin Heidelberg 2014

\begin{abstract}
We study the forward price dynamics in commodity markets realised as a process with values in a Hilbert space of absolutely continuous functions defined by Filipović (Consistency problems for Heath-Jarrow-Morton interest rate models, 2001). The forward dynamics are defined as the mild solution of a certain stochastic partial differential equation driven by an infinite-dimensional Lévy process. It is shown that the associated spot price dynamics can be expressed as a sum of OrnsteinUhlenbeck processes, or more generally, as a sum of certain stationary processes. These results link the possibly infinite-dimensional forward dynamics to classical commodity spot models. We continue with a detailed analysis of multiplication and integral operators on the Hilbert spaces and show that Hilbert-Schmidt operators are essentially integral operators. The covariance operator of the Lévy process driving the forward dynamics and the diffusion term can both be specified in terms of such operators, and we analyse in several examples the consequences on model dynamics and their probabilistic properties. Also, we represent the forward price for contracts delivering over a period in terms of an integral operator, a case being relevant for power and gas markets. In several examples, we reduce our general model to existing commodity spot and forward dynamics.
\end{abstract}

Keywords Forward price - Infinite-dimensional stochastic processes · Lévy processes $\cdot$ Commodity markets $\cdot$ Heath-Jarrow-Morton approach

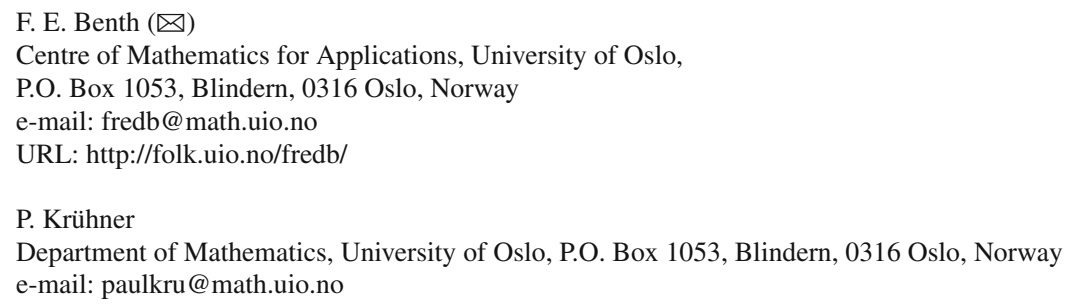


Mathematics Subject Classification (2010) $\quad 60 \mathrm{H} 15 \cdot 46 \mathrm{E} 35 \cdot 91 \mathrm{G} 80$

\section{Introduction}

In this paper, we analyse the dynamics of forward prices in commodity markets in the context of infinite-dimensional stochastic calculus. The motivation for our studies comes mainly from energy markets like power and gas where one finds strong seasonal patterns, a high degree of idiosyncratic risk over different market segments as well as non-Gaussian features like spikes.

In mathematical finance, the arbitrage-free forward price can be derived from the buy-and-hold strategy in the underlying spot commodity (see [32]). The forward price dynamics is thus implied from a given stochastic model of the spot commodity. If one specifies the price of the spot commodity by a semimartingale dynamics, one can represent the forward price as the conditional expected value of the spot at time of delivery, where the expectation is calculated with respect to an equivalent martingale measure $Q$. However, electricity is non-storable and hence not tradeable in the classical sense. Consequently, the buy-and-hold hedging strategy breaks down, and the spot price process does not need to be a $Q$-martingale itself. Hence, any equivalent measure $Q$ can be used as a pricing measure when deriving an arbitrage-free forward price. This raises the question of finding a financially valid $Q$.

As the fundamental relationship between the spot and forward is highly delicate in energy markets, it seems like a reasonable alternative to model the forward price dynamics directly. The alternative approach of modelling derivatives directly was first advocated for interest rate markets in Heath et al. [31], commonly referred to as the HJM approach, and the idea of this novel method has been transferred to other markets, see e.g., Carmona and Nadtochiy [19], Kallsen and Krühner [35] and Bühler [17].

Due to its mathematical nature, it is straightforward to transfer the HJM approach to commodity forward markets. This was first done by Clewlow and Strickland [20], and later analysed in the context of power markets by Benth and Koekebakker [11]. However, both these works used forward curve models driven by finite-dimensional Wiener noise (in fact, one-dimensional!). There are ample empirical evidences for a high degree of idiosyncratic risk in power forward markets, in particular. Andresen et al. [1] studied the Nordic power market NordPool, and found a clear correlation structure between contracts with different time to delivery. Earlier studies of the same market by Koekebakker and Ollmar [36] using principal component analysis also point towards a very high dimension of the noise. Moreover, marginal price behaviour is strongly non-Gaussian, as the study of Frestad [27] clearly indicates. These insights from real market prices motivate an HJM approach based on infinite-dimensional non-Gaussian noise.

The function spaces introduced by Filipović [25] provide a natural framework to model the forward curve evolution. These spaces have been applied in fixed-income markets for the forward rate dynamics, cf. [18]. Denoting by $F(t, T)$, the forward price at time $t \leq T$ for a contract delivering a spot commodity at time $T$, the HJM approach gives the dynamics of $F$ as the solution of a stochastic differential equation 


$$
\mathrm{d} F(t, T)=\beta(t, T) \mathrm{d} t+\Psi(t, T) \mathrm{d} L(t)
$$

for some infinite-dimensional Lévy process $L$ and appropriately defined parameters $\beta$ and $\Psi$. Since the forward curve $F(t, T)_{T \geq t}$ changes, its domain over time is more convenient to work with the Musiela parametrisation

$$
f(t, x):=F(t, t+x), \quad x \geq 0 .
$$

We interpret $x=T-t$ as time to delivery, whereas $T$ is time of delivery. Then, heuristically speaking, the forward curve follows the stochastic partial differential equation (SPDE)

$$
\mathrm{d} f(t, x)=\left(\beta(t, t+x)+\partial_{x} f(t, x)\right) \mathrm{d} t+\Psi(t, t+x) \mathrm{d} L(t) .
$$

However, in order to make sense of this SPDE, it is useful to work in an appropriate space of functions which contain the entire forward curve $(f(t, x))_{x \geq 0}$ for any time $t \geq 0$. For this purpose, we shall use the spaces introduced by Filipović [25].

We remark that the spot price of the commodity is recovered by passing to the limit for $x \downarrow 0$ in $f(t, x)$. Moreover, if we model the forward curve directly under the pricing measure $Q$ which is the common strategy in the HJM approach, martingale conditions must be imposed in the dynamics. It is to be noted that the forward contracts are liquidly traded financial assets in most commodity markets, and therefore it may sometimes be natural to model the dynamics under the objective (market) probability $P$.

In this paper, we provide a detailed analysis of the forward curve dynamics. In particular, we are able to derive various representations of the joint forward price dynamics for a given finite set of contracts, and relate this to finite-dimensional models. More specifically, we recover the covariance structure from the covariance operator of the infinite-dimensional noise of the initially given forward curve dynamics. Furthermore, we prove that the spot price dynamics in special cases can be represented as a sum of Ornstein-Uhlenbeck processes. Such mean-reverting processes are popular in commodity markets to model the stationary evolution of prices, reflecting the direct influence of demand and supply (see Benth et al. [8] for a discussion and references). More generally, we obtain a spot price dynamics in terms of so-called Lévy semistationary processes, which have gained recent attention in power markets (see Garcia et al. [28] for the special case of CARMA processes, and Barndorff-Nielsen et al. [4]). These processes are also natural in modelling the temperature dynamics, and thus are relevant to weather forward prices.

We also make a detailed study of operators on the spaces introduced by Filipović [25]. We have a particular attention on multiplication and integral operators, as these are natural objects when specifying $\Psi$ in (1), and when defining the covariance operator associated with (square-integrable) infinite-dimensional Lévy processes. We give complete characterisations of these operators, where we specifically show that all Hilbert-Schmidt operators essentially are integral operators. In examples, we link these operators to concrete models relevant for the forward dynamics in energy markets. 
In power and gas, and in fact also weather derivative markets, the forward contracts are typically settled over a delivery period and not at a specific delivery time. For example, in the NordPool market, forward contracts are settled financially on the hourly spot price over different delivery periods that range from a day to a year. As an application of our study of operators, we identify forward contracts with a delivery period as an operator on elements in the space defined by Filipović [25] mapping onto itself. Hence, the analytic properties of a fixed-delivery forward curve can be transported to a delivery-period forward curve by an operator, being in fact a sum of the identity operator and an explicitly given integral operator.

Our results are presented as follows: In the next section, we find various representations for stochastic processes which are linearly mapped to finite-dimensional spaces. Section three contains our main discussion on forward curve models for commodity markets where we derive various representations for the resulting spot price process, the correlation of forward price process and for the forward curve itself. In the last section, we analyse integral operators, Hilbert Schmidt operators and multiplication operators on the space defined by Filipović [25] in detail.

\subsection{Notation}

$\mathbb{R}$, resp. $\mathbb{C}$, denotes the real, resp. the complex numbers, and $\mathbb{R}_{+}:=[0, \infty)$ (resp. $\mathbb{R}_{-}:=(-\infty, 0]$ ) the non-negative (resp. non-positive) real numbers. $\left(\Omega,\left(\mathcal{F}_{t}\right)_{t \in \mathbb{R}_{+}}, \mathfrak{A}, P\right)$ will always denote a right-continuous filtered probability space. We denote the set of $p$-integrable functions from a measure space $(\Sigma, \mathcal{C}, \mu)$ to $\mathbb{R}$ by $L^{p}((\Sigma, \mu))$ and if there is no ambiguity with the measure, then we simply write $L^{p}(\Sigma)$ instead. For an operator $\mathcal{T} \in L\left(H_{1}, H_{2}\right), L\left(H_{1}, H_{2}\right)$ being the space of linear operators between the two Hilbert spaces $H_{1}$ and $H_{2}$, we denote the dual operator of $\mathcal{T}$ by $\mathcal{T}^{*}$. The set of positive operators with finite trace on a Hilbert space $H$ is denote by $L_{1}^{+}(H)$. For separable Hilbert spaces $U, H$ and an $U$-valued square integrable Lévy process $L$ with martingale covariance $\mathcal{Q}$ relative to some filtered probability space we denote by $\mathcal{L}_{L, T}^{2}(H)$ the set of predictable $L(U, H)$-valued processes such that

$$
\left.\int_{0}^{T} \operatorname{Tr}\left(\Psi(s) \mathcal{Q} \Psi(s)^{*}\right)\right) \mathrm{d} s<\infty, \quad t \geq 0,
$$

and $\mathcal{L}_{L}^{2}(H):=\bigcap\left\{\mathcal{L}_{L, T}^{2}(H): T>0\right\}$, cf. Peszat and Zabczyk [40, formula (8.6)] where Tr denotes the trace of the operator. Further unintroduced notations are used as in Peszat and Zabczyk [40].

\section{Representation of Functionals of Stochastic Integrals in a Hilbert Space}

In this Section, we derive some general results on the representation of linear functionals of stochastic integrals in a Hilbert space. These results will be useful in our 
analysis of the forward curve dynamics in Sect. 3, but are interesting in their own right as well.

Let us consider a stochastic integral $Y$ of the form

$$
Y(t)=\int_{0}^{t} \Psi(s) \mathrm{d} W(s),
$$

for some Brownian motion $W$ with values in a separable Hilbert space $U$ and an integrable stochastic process $\Psi: \mathbb{R}_{+} \times U \mapsto H$, with $H$ a separable Hilbert space (see Peszat and Zabczyk [40] for conditions). Sometimes one is only interested in one-dimensional marginals, i.e., one is interested in

$$
X(t):=\mathcal{T}(Y(t)),
$$

where $\mathcal{T}$ is a continuous linear functional on the state space $H$ of $Y$. Then, of course, we have

$$
X(t)=\int_{0}^{t}(\mathcal{T} \circ \Psi(s)) \mathrm{d} W(s) .
$$

If $U$ is finite dimensional, it is well known that there is some standard (real-valued) Brownian motion $B$ and some Itô integrable stochastic process $\sigma$ such that

$$
X(t)=\int_{0}^{t} \sigma(s) \mathrm{d} B(s)
$$

The next Theorem shows that a similar representation holds if $U$ is any separable Hilbert space:

Theorem 2.1 Let $n \in \mathbb{N}$ and $H, U$ be separable Hilbert spaces. Let $W$ be a square integrable and mean zero $U$-valued Wiener process with covariance $\mathcal{Q} \in L_{1}^{+}$. Assume that $\operatorname{dim} \operatorname{ran}(\mathcal{Q}) \geq n$ and $\mathcal{Q}$ is positive definite. Let $\Psi \in \mathcal{L}_{W}^{2}(H), \mathcal{T} \in L\left(H, \mathbb{R}^{n}\right)$ and define

$$
X(t):=\mathcal{T}\left(\int_{0}^{t} \Psi(s) \mathrm{d} W(s)\right) .
$$

Then there is an n-dimensional standard Brownian motion B such that

$$
X(t)=\int_{0}^{t} \sigma(s) \mathrm{d} B(s)
$$


where $\sigma(s):=\left(\mathcal{T} \Psi(s) \mathcal{Q} \Psi(s)^{*} \mathcal{T}^{*}\right)^{1 / 2} \in \mathcal{L}_{B}^{2}\left(\mathbb{R}^{n}\right)$. If $\sigma(s)$ is invertible in $\mathbb{R}^{n \times n}$ for $\lambda \otimes P$-almost any $s \in \mathbb{R}$, then $\sigma^{-1} \in \mathcal{L}_{X}^{2}\left(\mathbb{R}^{n}\right)$ and

$$
B(t)=\int_{0}^{t}(\sigma(s))^{-1} \mathrm{~d} X(s)
$$

Proof Peszat and Zabczyk [40, Theorem 8.7(v)] yield

$$
X(t)=\int_{0}^{t} \Gamma(s) \mathrm{d} W(s)
$$

where $\Gamma(s):=\mathcal{T} \circ \Psi(s)$. Peszat and Zabczyk [40, Corollary 8.17] imply

$$
\langle\langle X, X\rangle\rangle_{t}=\int_{0}^{t} \Gamma(s) \mathcal{Q} \Gamma(s)^{*} \mathrm{~d} s .
$$

Corollary 4.24 yields that $X$ has a.s. continuous paths. The assumptions assure that there is an $n$-dimensional standard Brownian motion $B$ on the filtered probability space $\left(\Omega,\left(\mathcal{F}_{t}\right)_{t \in \mathbb{R}_{+}}, \mathfrak{A}, P\right)$. Thus, Jacod [33, Corollaire $\left.14.47(\mathrm{~b})\right]$ yields that there is an $n$-dimensional standard Brownian motion $B$ such that

$$
X(t)=\int_{0}^{t} \sigma(s) \mathrm{d} B(s)
$$

Now assume that $\sigma(s)$ is an invertible element of $\mathbb{R}^{n \times n}$ for $\lambda \otimes P$-almost any $s \in \mathbb{R}$, denote the matrix inverse of $\sigma(s)$ by $\gamma(s)$ and denote the identity matrix on $\mathbb{R}^{n}$ by $I_{n}$. The representation (2) and Peszat and Zabczyk [40, Definition 8.3, Theorem 8.7(iv)] yield that the martingale covariance $\mathcal{Q}^{X}$ of $X$ is given by

$$
\mathcal{Q}_{t}^{X}=1_{\left\{\operatorname{Tr}\left(\sigma(t) \sigma(t)^{*}\right) \neq 0\right\}} \frac{\sigma(t) \sigma(t)^{*}}{\operatorname{Tr}\left(\sigma(t) \sigma(t)^{*}\right)}, \quad t \geq 0 .
$$

Then we have

$$
\mathrm{E}\left(\int_{0}^{T} \operatorname{Tr}\left(\gamma(s) \mathcal{Q}_{s}^{X} \gamma(s)^{*}\right) \mathrm{d}\langle X, X\rangle(s)\right)=n T<\infty
$$

and hence $\gamma \in \mathcal{L}_{X}^{2}\left(\mathbb{R}^{n}\right)$ by definition, cf. Peszat and Zabczyk [40, p. 113]. 
Hence

$$
\begin{aligned}
B(t) & =\int_{0}^{t} \sigma^{-1}(s) \sigma(s) \mathrm{d} B(s) \\
& =\int_{0}^{t} \sigma^{-1}(s) \mathrm{d} X(s) .
\end{aligned}
$$

Remark that if $n=1$, then there is a stochastic process $\gamma:=(\mathcal{T} \Psi)^{*} 1$ such that $(\mathcal{T} \Psi) x=\langle\gamma, x\rangle$ for any $x \in U$. If the set

$$
\left\{(\omega, t) \in \Omega \times \mathbb{R}_{+}: \mathcal{Q} \gamma(\omega, t)=0\right\}
$$

is a $P \otimes \lambda$-null set, then $\varphi:=\frac{\langle\gamma, \cdot\rangle}{\langle\mathcal{Q} \gamma, \gamma\rangle^{1 / 2}} \in \mathcal{L}_{W}^{2}(U)$ and the standard Brownian motion given in the theorem above is also given by

$$
B(t)=\int_{0}^{t} \varphi(s) \mathrm{d} W(s)
$$

Moreover, then we have

$$
\mathcal{T}\left(\int_{0}^{t} \Psi(s) \mathrm{d} W(s)\right)=\int_{0}^{t}\langle\gamma(s), \mathrm{d} W(s)\rangle=\int_{0}^{t}\langle\mathcal{Q} \gamma(s), \gamma(s)\rangle^{1 / 2} \mathrm{~d} B(s) .
$$

We extend the result in Theorem 2.1 to the class of Lévy processes defined as subordinated Brownian motions in the next Subsection.

\subsection{The Case of Subordinated Brownian Motion}

Let us recall from Benth and Krühner [12, Theorem 4.7] the definition of a subordinated Brownian motion:

Definition 2.2 A subordinated Brownian motion $L$ with values in some Hilbert space $U$ is a Lévy process such that there is a $U$-valued Brownian motion $W$ and a subordinator $\Theta$ which is independent of $W$ such that

$$
L(t)=W(\Theta(t)) .
$$

Subordinated Brownian motions $L, N$ are of the same type if there are Brownian motions $W_{L}, W_{N}$ and subordinators $\Theta_{L}, \Theta_{N}$ such that $W_{N}, \Theta_{N}$ are independent, $W_{L}, \Theta_{L}$ are independent, $\Theta_{L}, \Theta_{N}$ have the same law and 


$$
\begin{aligned}
L(t) & =W_{L}\left(\Theta_{L}(t)\right), \\
N(t) & =W_{N}\left(\Theta_{N}(t)\right)
\end{aligned}
$$

for any $t \in \mathbb{R}_{+}$.

Subordinated Brownian motions have some similarities with Brownian motion if the subordinator has finite first moment. In particular, the set of integrands can be compared easily. First, we recall the notion of time-changed filtrations:

Definition 2.3 A time change is a right-continuous increasing family $(\theta(t))_{t \in \mathbb{R}_{+}}$of stopping times with respect to some right-continuous filtration $\left(\mathcal{F}_{t}\right)_{t \in \mathbb{R}_{+}}$. The time changed filtration is the filtration given by

$$
\mathcal{F}_{t}^{\theta}:=\mathcal{F}_{\theta(t)}=\bigcap_{s>t} \mathcal{F}_{\theta(s)} \quad t \in \mathbb{R}_{+}
$$

Let $X$ be an $\mathcal{F}$-adapted stochastic process and assume that the time-change is finite valued. The time-changed process is given by $X^{\theta}(t):=X(\theta(t)), t \in \mathbb{R}_{+}$.

We have the following result on stochastic integration with respect to subordinated Brownian motions.

Lemma 2.4 Let $W$ be a mean-zero Brownian motion with values in a separable Hilbert space $U$ relative to some filtration $\left(\mathcal{F}_{t}\right)_{t \in \mathbb{R}_{+}}, H$ be another separable Hilbert space, $\psi \in \mathcal{L}_{L}^{2}(\mathcal{G}, H)$, $\Theta$ be a non-zero subordinator with finite moment such that $\Theta(t)$ is a stopping time for each $t \in \mathbb{R}_{+}$. Let $\left(\mathcal{G}_{t}\right)_{t \in \mathbb{R}_{+}}$be the time-changed filtration given by $\mathcal{G}_{t}:=\mathcal{F}_{\Theta(t)}$ and $L(t):=W(\Theta(t)), t \in \mathbb{R}_{+}$. Then $L$ is a $U$-valued square integrable Lévy process and there is an isometric embedding

$$
\Gamma: \mathcal{L}_{L}^{2}(\mathcal{G}, H) \rightarrow \mathcal{L}_{W}^{2}(\mathcal{F}, H)
$$

such that

$$
\int_{0}^{t} \psi(s) \mathrm{d} L(s)=\int_{0}^{\Theta(t)} \Gamma(\psi)(s) \mathrm{d} W(s) \quad P \text {-a.s. },
$$

where the left stochastic integral is with respect to the filtration $\left(\mathcal{G}_{t}\right)_{t \in \mathbb{R}_{+}}$, the right stochastic integral is with respect to the filtration $\left(\mathcal{F}_{t}\right)_{t \in \mathbb{R}_{+}}$.

Proof $\Theta$ is a time-change on the filtration $\left(\mathcal{F}_{t}\right)_{t \in \mathbb{R}_{+}}$by definition. Benth and Krühner [12, Theorem 4.7] yield that $L$ is a Lévy process in its own right, however its increments are independent of the time-changed filtration and thus it is Lévy relative to the filtration $\left(\mathcal{G}_{t}\right)_{t \in \mathbb{R}_{+}}$. In order to establish the isometric embedding $\Gamma$, it is sufficient to work with elementary integrands. Let $\psi \in \mathcal{L}_{L}^{2}(\mathcal{G}, H)$ be elementary, i.e., there is $n \in \mathbb{N}, 0 \leq a_{j} \leq b_{j}<\infty, \mathcal{G}_{a_{j}}$-measurable square integrable random variables $Y_{j}$ and $\varphi_{j} \in L(U, H)$ such that

$$
\psi=\sum_{j=1}^{n} Y_{j} 1_{] a_{j}, b_{j}\right]} \varphi_{j}
$$


Define $\psi_{\Theta}:=\sum_{j=1}^{n} Y_{j} 1_{\left.1 \Theta\left(a_{j}\right), \Theta\left(b_{j}\right)\right]} \varphi_{j}$. Observe that $\psi_{\Theta}$ does not depend on the representation of $\psi$. We have $\psi_{\Theta} \in \mathcal{L}_{W}^{2}(\mathcal{F}, H)$ and

$$
\begin{aligned}
\int_{0}^{t} \psi(s) \mathrm{d} L(s) & =\sum_{j=1}^{n} Y_{j} \varphi_{j}\left(L\left(t \wedge b_{j}\right)-L\left(t \wedge a_{j}\right)\right) \\
& =\sum_{j=1}^{n} Y_{j} \varphi_{j}\left(W\left(\Theta(t) \wedge \Theta\left(b_{j}\right)\right)-W\left(\Theta(t) \wedge \Theta\left(a_{j}\right)\right)\right) \\
& =\int_{0}^{\Theta(t)} \psi_{\Theta}(s) \mathrm{d} W(s) .
\end{aligned}
$$

Moreover, we have

$$
\begin{aligned}
\int_{0}^{\infty} \mathrm{E}\left(\left\langle\mathcal{Q}^{L} \psi(s), \psi(s)\right\rangle\right) \mathrm{d}\langle L, L\rangle(s) & =\mathrm{E}\left(\left(\int_{0}^{\infty} \psi(s) \mathrm{d} L(s)\right)^{2}\right) \\
& =\mathrm{E}\left(\left(\lim _{n \rightarrow \infty} \int_{0}^{\Theta(n)} \psi_{\Theta}(s) \mathrm{d} W(s)\right)^{2}\right) \\
& =\mathrm{E}\left(\left(\int_{0}^{\infty} \psi_{\Theta}(s) \mathrm{d} W(s)\right)^{2}\right) \\
& =\int_{0}^{\infty} \mathrm{E}\left(\left\langle\mathcal{Q}^{W} \psi_{\Theta}(s), \psi_{\Theta}(s)\right\rangle\right) \mathrm{d}\langle W, W\rangle(s),
\end{aligned}
$$

where $\mathcal{Q}^{L}$ denotes the martingale covariance of $L$ and $\mathcal{Q}^{W}$ denotes the martingale covariance of $W$.

From the construction, we can see that Eq. (3) holds for elementary integrands, and thus for all integrands by a density argument.

By appealing to this Lemma, we can derive a connection between functionals of the infinite-dimensional stochastic integral and finite-dimensional versions of it.

Theorem 2.5 Let $n \in \mathbb{N}$ and $H, U$ be separable Hilbert spaces. Let L be a non-zero, square integrable mean zero $U$-valued subordinated Brownian motion with martingale covariance $\mathcal{Q} \in L_{1}^{+}$. Assume that $\operatorname{dim} \operatorname{ran}(\mathcal{Q}) \geq n$. Let $\Psi \in \mathcal{L}_{L}^{2}(H), \mathcal{T} \in L\left(H, \mathbb{R}^{n}\right)$ and define 


$$
X(t):=\mathcal{T}\left(\int_{0}^{t} \Psi(s) \mathrm{d} L(s)\right)
$$

Then there is an n-dimensional square integrable mean-zero Lévy process $N$ such that

$$
X(t)=\int_{0}^{t} \sigma(s) \mathrm{d} N(s)
$$

where $\sigma(s):=\left(\mathcal{T} \Psi(s) \mathcal{Q} \Psi(s)^{*} \mathcal{T}^{*}\right)^{1 / 2} \in \mathcal{L}_{N}^{2}\left(\mathbb{R}^{n}\right)$. If $\sigma(s)$ is invertible in $\mathbb{R}^{n \times n}$ for $\lambda \otimes P$-almost any $s \in \mathbb{R}$, then $\sigma^{-1} \in \mathcal{L}_{X}^{2}\left(\mathbb{R}^{n}\right)$ and

$$
N(t)=\int_{0}^{t}(\sigma(s))^{-1} \mathrm{~d} X(s) .
$$

Proof First we assume that $\Psi$ is elementary, i.e., there are $n \in \mathbb{N}, 0 \leq a_{j} \leq b_{j}<\infty$, $\mathcal{F}_{a_{j}}$-measurable square integrable random variabes $Y_{j}$ and $\varphi_{j} \in L(U, H)$ such that

$$
\Psi=\sum_{j=1}^{n} Y_{j} 1_{] a_{j}, b_{j}\right]} \varphi_{j} .
$$

By definition of $L$, we have $L(t)=W(\Theta(t))$ for a $U$-valued Wiener process $W$ with martingale covariance $\mathcal{Q}^{W}$ and a subordinator $\Theta$. Let $\Gamma$ be the isometric embedding given in Lemma 2.4. Note that $\Gamma(\mathcal{T} \Psi)=\mathcal{T} \Gamma(\Psi)$ because this holds if $\Psi$ is elementary. Peszat and Zabczyk [40, Theorem 8.7(v)], Lemma 2.4 and Theorem 2.1 yield

$$
\begin{aligned}
\mathcal{T}\left(\int_{0}^{t} \Psi(s) \mathrm{d} L(s)\right) & =\int_{0}^{t} \mathcal{T} \Psi(s) \mathrm{d} L(s) \\
& =\int_{0}^{\Theta(t)} \Gamma(\mathcal{T} \Psi)(s) \mathrm{d} W(s) \\
& =\int_{0}^{\Theta(t)} \mathcal{T} \Gamma(\Psi)(s) \mathrm{d} W(s) \\
& =\int_{0}^{\Theta(t)} \sigma_{1}(s) \mathrm{d} B(s),
\end{aligned}
$$


where $B$ is a standard Brownian motion on $\mathbb{R}^{n}$ and

$$
\begin{aligned}
\sigma_{1}(t) & =\left(\mathcal{T} \Gamma(\Psi)(t) \mathcal{Q}^{W} \Gamma(\Psi)^{*}(t) \mathcal{T}^{*}\right)^{1 / 2} \\
& =\Gamma\left(\left(\mathcal{T} \Psi \mathcal{Q}^{W} \Psi^{*} \mathcal{T}^{*}\right)^{1 / 2}\right)(t) .
\end{aligned}
$$

Applying Lemma 2.4 again yields

$$
\mathcal{T}\left(\int_{0}^{t} \Psi(s) \mathrm{d} L(s)\right)=\int_{0}^{t} \sigma(s) \mathrm{d} N(s)
$$

where $N(t):=B(\Theta(t)), t \in \mathbb{R}_{+}$and

$$
\sigma(t)=\left(\mathcal{T} \Psi(t) \mathcal{Q}^{W} \Psi(t)^{*} \mathcal{T}^{*}\right)^{1 / 2}
$$

Sato [43, Theorem 30.1] implies that $N$ is an $n$-dimensional Lévy process with the desired properties. Observe that the martingale covariance $\mathcal{Q}^{L}$ of $L$ and $\mathcal{Q}^{W}$ coincide.

The general case can be deduced by a density argument.

We consider an example: Let $\Theta$ be an inverse Gaussian subordinator, that is, a Lévy process with increasing paths where $\Theta(1)$ is inverse Gaussian distributed. Then, $L(t)=W(\Theta(t))$ is a Hilbert-space valued normal inverse Gaussian (HNIG) Lévy process in the sense of Benth and Krühner [12, Definition 4.1]. The Lévy process $N$ in the theorem above becomes an $n$-variate normal inverse Gaussian (MNIG) Lévy process (see Rydberg [42]). In particular, in the case $n=1$, it is an ordinary NIG Lévy process (see Barndorff-Nielsen [3]). NIG Lévy processes are frequently used for modelling asset prices in various financial markets, including commodities (see Börger et al. [15]) and power (Andresen et al. [1], and Benth et al. [27]). HNIG Lévy processes are relevant in the context of modelling the dynamics of the power forward curve.

After these general considerations, we shall now focus on the dynamics of forward prices and various representations in the remainder of the paper.

\section{Forward Curve Modelling and Representations}

We are interested in studying the dynamics of the forward price $F(t, T), 0 \leq t \leq T$, of a contract delivering a commodity at time $T>0$. We shall mostly be concerned with the more convenient representation of $F$ in terms of the Musiela parametrisation, where we let

$$
F(t, T)=f(t, T-t)
$$


for a function $f(t, x), x \geq 0$, denoting the forward price at time $t$ for a contract with time to delivery $x$. We shall interpret $f$ as a stochastic process with values in a space of functions on $\mathbb{R}_{+}$.

More specifically, we consider the Hilbert spaces $H_{w}$ introduced by Filipović [25, Chap. 5]. These Hilbert-spaces are suitable for modelling the forward rate curves in the Musiela parametrisation, see e.g., Filipović et al. [26]. As the modelling of forward prices are similar in idea to the context of forward rates in fixed-income markets, we adopt the spaces $H_{w}$ for our analysis.

\subsection{Some Preliminary Results on $H_{w}$}

Let us introduce and recall some basic facts of the spaces $H_{w}$ before turning our attention to forward curves and their dynamics. We remark that the following results on $H_{w}$ can be essentially found in Filipović [25, Chaps. 4 and 5]. However, some of the results are slight modifications where proofs have been added.

Let $\mathrm{AC}\left(\mathbb{R}_{+}, \mathbb{R}\right)$ be the set of functions $g: \mathbb{R}_{+} \rightarrow \mathbb{R}$ which are absolutely continuous. For a function $w: \mathbb{R}_{+} \rightarrow[1, \infty)$ being continuous, increasing and $w(0)=1$, define

$$
H_{w}:=\left\{g \in \mathrm{AC}\left(\mathbb{R}_{+}, \mathbb{R}\right): \int_{0}^{\infty} w(x) g^{\prime}(x)^{2} \mathrm{~d} x<\infty\right\}
$$

We introduce the inner product $\langle g, h\rangle:=g(0) h(0)+\int_{0}^{\infty} w(x) g^{\prime}(x) h^{\prime}(x) \mathrm{d} x$ and the corresponding norm $\|g\|_{w}:=\sqrt{\langle g, g\rangle}$ for any $g, h \in H_{w}$. Note that all the following results also hold if $\mathrm{AC}\left(\mathbb{R}_{+}, \mathbb{R}\right)$ is replaced by $\mathrm{AC}\left(\mathbb{R}_{+}, \mathbb{C}\right)$ which, however, has no financial meaning.

We observe that the point evaluation $\delta_{x}: H_{w} \rightarrow \mathbb{R}$ for any $x \in \mathbb{R}$, where $\delta_{x}(g)=$ $g(x)$, is a continuous linear functional on $H_{w}$. Its dual operator can be explicitly characterised, as is shown in the following Lemma:

Lemma 3.1 For $x \in \mathbb{R}$, define

$$
h_{x}: \mathbb{R}_{+} \rightarrow \mathbb{R}, y \mapsto 1+\int_{0}^{y \wedge x} \frac{1}{w(s)} \mathrm{d} s .
$$

Then the dual operator of $\delta_{x}$ is given by

$$
\delta_{x}^{*}: \mathbb{R} \rightarrow H_{w}, c \mapsto c h_{x}
$$

and its operator norm is given by

$$
\left\|\delta_{x}\right\|_{\mathrm{op}}^{2}=h_{x}(x)
$$


Proof The first statement is a special case of Filipović [25, Lemma 5.3.1]. Next, since the operator norm coincides with the norm of $h_{x}$ we get

$$
\left\|\delta_{x}\right\|_{\mathrm{op}}^{2}=h_{x}(0) h_{x}(0)+\int_{0}^{\infty} w(y)\left(h_{x}^{\prime}(y)\right)^{2} \mathrm{~d} y=1+\int_{0}^{x} \frac{1}{w(y)} \mathrm{d} y=h_{x}(x) .
$$

Hence, the Lemma follows.

Under the additional assumption

$$
\int_{0}^{\infty} \frac{1}{w(x)} \mathrm{d} x<\infty
$$

the functions in $H_{w}$ have a continuous continuation to infinity and the "point evaluation at infinity' corresponds to taking the scalar product with the function

$$
h_{\infty}: \mathbb{R}_{+} \rightarrow \mathbb{R}, x \mapsto 1+\int_{0}^{x} \frac{1}{w(s)} \mathrm{d} s
$$

These facts are summarised in the following Lemma.

Lemma 3.2 Assume that $\int_{0}^{\infty} \frac{1}{w(x)} \mathrm{d} x<\infty$. Then

$$
\|g\|_{\infty}:=\sup _{x \in \mathbb{R}_{+}}|g(x)| \leq c\|g\|_{w} .
$$

where $c:=\sqrt{1+\int_{0}^{\infty} \frac{1}{w(x)} \mathrm{d} x}$. Moreover, $h_{\infty} \in H_{w}$ and

$$
\lim _{x \rightarrow \infty} g(x)=\left\langle h_{\infty}, g\right\rangle
$$

for any $g \in H_{w}$ and $h_{\infty}$ defined in (4).

Proof We have

$$
\left\|h_{\infty}\right\|_{w}^{2}=1+\int_{0}^{\infty} \frac{1}{w(x)} \mathrm{d} x=c^{2}
$$

Hence $h_{\infty} \in H_{w}$. With $h_{x}$ given in Lemma 3.1 we see that

$$
\left\|h_{\infty}-h_{x}\right\|_{w}^{2}=\int_{x}^{\infty} \frac{1}{w(y)} \mathrm{d} y \rightarrow 0, \quad x \rightarrow \infty .
$$


Thus, $h_{x} \rightarrow h_{\infty}$ in $H_{w}$ for $x \rightarrow \infty$. Thus, Lemma 3.1 implies

$$
\lim _{x \rightarrow \infty} g(x)=\lim _{x \rightarrow \infty}\left\langle h_{x}, g\right\rangle=\left\langle h_{\infty}, g\right\rangle
$$

In particular, $g$ is bounded. Let $x \in \mathbb{R}_{+}$. Then Lemma 3.1 yields

$$
|g(x)|^{2} \leq\left\|h_{x}\right\|_{w}^{2}\|g\|_{w}^{2}=h_{x}^{2}(x)\|g\|_{w}^{2} \leq c^{2}\|g\|_{w}^{2},
$$

and hence $\|g\|_{\infty} \leq c\|g\|_{w}$.

The following technical Lemma is needed later. It shows that the square function is Lipschitz-continuous on bounded sets.

Lemma 3.3 Assume that $k:=\int_{0}^{\infty} \frac{1}{w(x)} d x<\infty$. Then

$$
\left\|g_{1}^{2}-g_{2}^{2}\right\|_{w} \leq \sqrt{5+4 k^{2}}\left\|g_{1}+g_{2}\right\|_{w}\left\|g_{1}-g_{2}\right\|_{w}
$$

for any $g_{1}, g_{2} \in H_{w}$. In particular,

$$
(\cdot)^{2}: H_{w} \rightarrow H_{w}, g \mapsto g^{2}
$$

is Lipschitz-continuous on bounded subsets of $H_{w}$.

Clearly, the second part follows from the first. Since a direct proof of the first part is very technical, we delegate it to Corollary 4.19 below where the Lipschitzcontinuity follows easily from the Banach algebra structure of $H_{w}$ relative to pointwise multiplication (see Prop. 4.18).

In the definition of the dynamics of $f$, the forward price, the differential operator with respect to $x, \partial_{x}$, naturally occurs. We have the following convenient result for this operator on $H_{w}$ :

Theorem 3.4 We have

$$
\partial_{x}:\left\{g \in H_{w}: g^{\prime} \in H_{w}\right\} \rightarrow H_{w}, g \mapsto g^{\prime}
$$

which is the generator of the strongly continuous semigroup given by

$$
\mathcal{U}_{t} g(x)=g(t+x)
$$

for any $t, x \in \mathbb{R}_{+}, g \in H_{w}$.

Proof See Filipović [25, Theorem 5.1.1, Remark 5.1.1].

The method of the moving frame works especially well with quasi-contractive generators of strongly continuous semigroups, cf. Tappe [45]. 
Lemma 3.5 The semigroup $\mathcal{U}_{t}$ generated by $\partial_{x}$ is quasi-contractive, i.e., there is a constant $\beta>0$ such that

$$
\left\|\mathcal{U}_{t}\right\|_{\text {op }} \leq e^{t \beta}
$$

for any $t>0$.

Proof Let $g \in H_{w}$ such that $\|g\|_{w} \leq 1$ and $t \in[0,1]$. Then

$$
\begin{aligned}
\left\|\mathcal{U}_{t} g\right\|_{w}^{2} & =g^{2}(t)+\int_{0}^{\infty}\left(g^{\prime}(t+y)\right)^{2} w(y) \mathrm{d} y \\
& \leq g^{2}(0)+2 g(0) \int_{0}^{t} g^{\prime}(y) \mathrm{d} y+\left(\int_{0}^{t} g^{\prime}(y) \mathrm{d} y\right)^{2}+\int_{t}^{\infty}\left(g^{\prime}(y)\right)^{2} w(y) \mathrm{d} y \\
& \leq g^{2}(0)+2\|g\|_{w} \mid\left\langle g, h_{t}-h_{0}\right\rangle+t \int_{0}^{t}\left(g^{\prime}(y)\right)^{2} \mathrm{~d} y+\int_{t}^{\infty}\left(g^{\prime}(y)\right)^{2} w(y) \mathrm{d} y \\
& \leq g^{2}(0)+2\|g\|_{w}^{2}\left\|h_{t}-h_{0}\right\|_{w}+\int_{0}^{\infty}\left(g^{\prime}(y)\right)^{2} w(y) \mathrm{d} y \\
& =\|g\|_{w}^{2}\left(1+2\left\|h_{t}-h_{0}\right\|_{w}\right),
\end{aligned}
$$

where $h_{t}$ is defined as in Lemma 3.1. Above we used the Schwarz inequality for the second inequality and the Cauchy-Schwarz inequality for the third inequality. Thus, we have

$$
\left\|\mathcal{U}_{t}\right\|_{\text {op }} \leq \sqrt{1+2\left\|h_{t}-h_{0}\right\|_{w}} \leq 1+t \leq \exp (t)
$$

which shows the asserted inequality for $t \in[0,1]$. The inequality for $t>1$ follows by iteration.

With these results on the space $H_{w}$ at hand, we are now ready to start our analysis of the forward dynamics.

\subsection{General Forward Curve Dynamics}

We consider a general stochastic equation of the form

$$
\mathrm{d} f(t)=\left(\partial_{x} f(t)+\beta(t)\right) \mathrm{d} t+\Psi(t) \mathrm{d} L(t), \quad t \geq 0
$$


in the sense of its mild formulation

$$
f(t)=\mathcal{U}_{t} f_{0}+\int_{0}^{t} \mathcal{U}_{t-s} \beta(s) \mathrm{d} s+\int_{0}^{t} \mathcal{U}_{t-s} \Psi(s) \mathrm{d} L(s), \quad t \geq 0
$$

to model the dynamics of the forward price specified in the Musiela parametrisation. Here, $\left(\mathcal{U}_{t}\right)_{t \geq 0}$ denotes the semigroup generated by $\partial_{x}$, cf. Lemma 3.5, and we assume $L$ to be a square-integrable mean-zero Lévy process with values in some Hilbert space $U$ and $\mathcal{Q}$ its covariance operator. Furthermore, we denote by $f(0)=f_{0}$ the initial condition, where $f_{0} \in H_{w}$. The 'volatility' of the forward dynamics is $\Psi \in \mathcal{L}_{L}^{2}\left(H_{w}\right)$, and we have the integrable drift $\beta: \mathbb{R}_{+} \times \Omega \rightarrow H_{w}$. For the space $H_{w}$, we assume that $k^{2}:=\int_{0}^{\infty} \frac{1}{w(s)} \mathrm{d} s<\infty$.

Remark 3.6 If $(b, C, F)$ is the characteristics of $L$ relative to some truncation function $\chi$, then the mean $m$ and the covariance operator $\mathcal{Q}$ of $L(1)$ are given by

$$
\begin{gathered}
\mathcal{Q} x=C x+\int_{U} y\langle x, y\rangle F(\mathrm{~d} y) \text { and } \\
m=b+\int_{U}(y-\chi(y)) F(\mathrm{~d} y)
\end{gathered}
$$

for any $x \in U$, cf. [40, Definition 4.45], the angle bracket is given by $\langle L, L\rangle_{t}=t \operatorname{Tr}(\mathcal{Q})$ (cf. [40, p. 35]), the operator angle bracket by $\langle\langle L, L\rangle\rangle_{t}=t \mathcal{Q}$ (cf. [40, Theorem 8.2]) and hence the martingale covariance by $\mathcal{Q} / \operatorname{Tr}(\mathcal{Q})$ (cf. [40, Definition 8.3]).

A natural way to set up an equation like (5) is to consider stochastic partial differential equations, i.e., to assume that $\beta(t)=a(t, f(t)), \Psi(t)=\psi(t, f(t))$ for some suitable functions $a, \psi$. The following statement is about existence and uniqueness of mild solutions to (5) needed for this paper under such a state-dependent specification of $\beta$ and $\psi$.

Proposition 3.7 Let $a: \mathbb{R}_{+} \times H_{w} \rightarrow H_{w}, \psi: \mathbb{R}_{+} \times H_{w} \rightarrow L\left(U, H_{w}\right)$ such that there is an increasing function $K: \mathbb{R}_{+} \rightarrow \mathbb{R}_{+}$such that the following Lipschitz conditions hold.

$$
\begin{aligned}
\|a(t, g)-a(t, h)\|_{w} & \leq K(t)\|g-h\|_{w}, \quad g, h \in H_{w}, t \in \mathbb{R}_{+}, \\
\|a(t, g)\|_{w} & \leq K(t)\left(1+\|g\|_{w}\right), \quad t \in, g \in H_{w}, \mathbb{R}_{+}, \\
\|\psi(t, g)-\psi(t, h)\|_{\mathrm{op}} & \leq K(t)\|g-h\|_{w}, \quad g, h \in H_{w}, t \in \mathbb{R}_{+}, \\
\|\psi(t, g)\|_{\mathrm{op}} & \leq K(t)\left(1+\|g\|_{w}\right), \quad g \in H_{w}, t \in \mathbb{R}_{+},
\end{aligned}
$$

and let $f_{0} \in H_{w}$. Then there is a unique adapted $H_{w}$-valued càdlàg stochastic process $f$ satisfying 


$$
f(t):=\mathcal{U}_{t} f_{0}+\int_{0}^{t} \mathcal{U}_{t-s} a(s, f(s)) \mathrm{d} s+\int_{0}^{t} \mathcal{U}_{t-s} \psi(s, f(s)) \mathrm{d} L(s), \quad t \geq 0,
$$

being the mild solution to

$$
\mathrm{d} f(t)=\left(\partial_{x} f(t)+a(t, f(t))\right) \mathrm{d} t+\psi(t, f(t)) \mathrm{d} L(t), \quad t \geq 0 .
$$

Proof Lemma 3.5 states that $\mathcal{U}_{t}$ is quasi-contractive. Thus, Tappe [45, Theorem 4.5] yields the claim.

We remark that the Lipschitz and growth condition listed above are considerably stronger than the conditions stated in Tappe [45, Theorem 4.5], but we have restricted our attention to such coefficients $a$ and $\psi$ for simplicity only. In the rest of the paper, we do not assume any functional representation for $\beta$ and $\Psi$ as in Proposition 3.7, but apply the representation (6) for $f$ from time to time. We refer to Peszat and Zabczyk [40] for a definition and further analysis of mild solutions to stochastic partial differential equations.

Before we start our discussion of the main topic let us discuss physical and risk neutral model perspectives. For simplicity, assume that the SPDE (5) is driven by a Wiener process $W$, i.e.,

$$
\mathrm{d} f(t)=\left(\partial_{x} f(t)+\beta(t)\right) \mathrm{d} t+\Psi(t) \mathrm{d} W(t), \quad t \geq 0 .
$$

The fundamental theorem of asset pricing states that there is an equivalent $\sigma$-martingale measure $Q \sim P$, cf. Delbaen and Schachermayer [21, Main theorem 1.1], for any market with finitely many securities. To avoid further complications, we simply assume the existence of a martingale measure $Q \sim P$. Then Carmona and Tehranchi [18, Theorem 4.2] yield that the $Q$-dynamics of $f$ are given by

$$
\mathrm{d} f(t)=\left(\partial_{x} f(t)+\left(\beta(t)-\mathcal{Q}^{1 / 2} \psi(t)\right)\right) \mathrm{d} t+\Psi(t) \mathrm{d} W(t), \quad t \geq 0
$$

for some $H_{w}$-valued predictable stochastic process $\psi$ where $\mathcal{Q}$ is the martingale covariance of $W$. However, the forwards $F(t, T)$ in delivery time parametrisation must be martingales under the martingale measure $Q$ and hence Theorem 3.9 below yields

$$
\beta(t)=\mathcal{Q}^{1 / 2} \psi(t), \quad t \geq 0 .
$$

We conclude that the no-arbitrage paradigm implies that the drift term $\beta$ must stay in the range of $\mathcal{Q}^{1 / 2}$. Of course, if the driving noise is not a Wiener process, then the analysis gets more complicated which we can already see in the finite-dimensional case, cf. Jacod and Shiryaev [34, Theorem III.3.24]. If we want to model under the risk neutral measure directly, then we can and must put $\beta=0$.

Note that we recover the spot price dynamics $S(t)$ by applying the evaluation map $\delta_{0}$ on $f$ (cf. Proposition 3.1):

$$
S(t):=\delta_{0}(f(t))
$$


We have the following result for the implied spot price dynamics from the forward price $f(t)$ in the case $L$ which is a subordinated Brownian motion in $U$ :

Theorem 3.8 If $L$ is a subordinated Brownian motion, i.e., $L(t)=W(\Theta(t)), t \geq 0$ for some $U$-valued Brownian motion $W$ and some subordinator $\Theta$, then

$$
S(t)=f_{0}(t)+\int_{0}^{t} \beta(s)(t-s) \mathrm{d} s+\int_{0}^{t} \sigma(t, s) \mathrm{d} N(s)
$$

for any $t \in \mathbb{R}_{+}$where $N(t)=B(\Theta(t)), t \geq 0$ and $B$ is a constant multiple of an $\mathbb{R}$-valued Brownian motion and

$$
\sigma^{2}(t, s)=\left\langle\Psi(s) \mathcal{Q} \Psi^{*}(s) h_{t-s}, h_{t-s}\right\rangle
$$

for $s, t \in \mathbb{R}_{+}$.

Proof Note that if $B$ is an $\mathbb{R}$-valued Brownian motion, $\varphi \in \mathcal{L}_{L}^{2}(\mathbb{R})$ is an operatorvalued predictable process, then the integral defined as in Peszat and Zabczyk [40] can be represented as a stochastic integral as in Jacod and Shiryaev [34] by

$$
\int_{0}^{t} \varphi(s) \mathrm{d} B(s)=\int_{0}^{t} \varphi(s)(1) \mathrm{d} B(s)
$$

and by the Itô isometry we have

$$
\mathrm{E}\left(\int_{0}^{t} \varphi(s) \mathrm{d} B(s)\right)^{2}=\int_{0}^{t} \operatorname{ETr}\left(\varphi(s) \varphi(s)^{*}\right) \mathrm{d} s=\int_{0}^{t} \mathrm{E}\left((\varphi(s)(1))^{2}\right) \mathrm{d} s .
$$

The definition of $S$ in (7) and the representation of $f$ in (6) yield

$$
S(t)=\delta_{0}(f(t))=f_{0}(t)+\int_{0}^{t} \beta(s)(t-s) \mathrm{d} s+\int_{0}^{t} \delta_{t-s} \Psi(s) \mathrm{d} L(s)
$$

for any $t \in \mathbb{R}_{+}$. Thus, applying Theorem 2.5 and Lemma 3.1 we find

$$
S(t)=f_{0}(t)+\int_{0}^{t} \beta(s)(t-s) \mathrm{d} s+\int_{0}^{t} \sigma(t, s) \mathrm{d} N(s)
$$

for any $t \in \mathbb{R}_{+}$. Here, $N$ is an $\mathbb{R}$-valued subordinated Brownian motion of the same type as $L$, and 


$$
\begin{aligned}
\sigma^{2}(t, s) & =\delta_{t-s} \Psi(s) \mathcal{Q} \Psi^{*}(s) \delta_{t-s}^{*} 1 \\
& =\left\langle\delta_{t-s} \Psi(s) \mathcal{Q} \Psi^{*}(s) h_{t-s}, 1\right\rangle \\
& =\left\langle\Psi(s) \mathcal{Q} \Psi^{*}(s) h_{t-s}, h_{t-s}\right\rangle
\end{aligned}
$$

for any $s, t \in \mathbb{R}_{+}$.

Let us for a moment assume that $\beta=0$ in Theorem 3.8. In the case, $\Psi$ is deterministic, $\sigma(t, s)$ will become deterministic as well. Hence, the spot price dynamics will be a Volterra process. If, additionally, $\Psi$ is constant, then

$$
\sigma^{2}(t, s)=\left\langle\Psi \mathcal{Q} \Psi^{*} h_{t-s}, h_{t-s}\right\rangle=: \gamma^{2}(t-s) .
$$

But then $S$ will be a so-called Lévy stationary process. An extension of Lévy stationary processes is so-called Lévy semistationary (LSS) processes. By choosing $\Psi(s)=$ $\widetilde{\sigma}(s) \mathcal{T}$ for some linear operator $\mathcal{T} \in L\left(U, H_{w}\right)$ and a predictable $\mathbb{R}$-valued stochastic $\widetilde{\sigma}$, then $S$ in Theorem 3.8 becomes an LSS process. Indeed, the volatility is in this case

$$
\sigma^{2}(t, s)=\widetilde{\sigma}^{2}(s)\left\langle\mathcal{T} \mathcal{Q} \mathcal{T}^{*} h_{t-s}, h_{t-s}\right\rangle
$$

Such processes have been applied to model not only the stochastic dynamics of energy prices (see Barndorff-Nielsen et al. [4]), but also other random phenomena like wind speed and temperature (see Benth and Šaltytė Benth [9]) and turbulence (see BarndorffNielsen and Schmiegel [5]). We may discuss similar specifications of the $\operatorname{drift} \beta$ in light of these different special classes of spot models.

We are interested in a similar result for the dynamics of the forward price $t \mapsto$ $F(t, T), t \leq T$, for a contract delivering at time $T$. Obviously,

$$
F(t, T):=\delta_{T-t} f(t)
$$

for $T \geq t \geq 0$. A natural adaption of the proof of Theorem 3.8 reveals an analogous representation of $F(t, T)$ as for the spot price $S(t)$. We present this result for the dynamics of the bivariate process $t \mapsto\left(F\left(t, T_{1}\right), F\left(t, T_{2}\right)\right)=\left(\delta_{T_{1}-t} f(t), \delta_{T_{2}-t} f(t)\right)$, with $0 \leq t \leq T_{1} \leq T_{2}<\infty$. This will not only provide us with the dynamics of a single contract, but also give information about how two forward contracts with different times of delivery are connected within our model.

Theorem 3.9 If $L$ is a subordinated Brownian motion, i.e., $L(t)=W(\Theta(t)), t \geq 0$ for some $U$-valued Brownian motion $W$ and some subordinator $\Theta$, then

$$
\left(\begin{array}{l}
F\left(t, T_{1}\right) \\
F\left(t, T_{2}\right)
\end{array}\right)=\left(\begin{array}{l}
f_{0}\left(T_{1}\right) \\
f_{0}\left(T_{2}\right)
\end{array}\right)+\int_{0}^{t}\left(\begin{array}{l}
\beta(s)\left(T_{1}-s\right) \\
\beta(s)\left(T_{2}-s\right)
\end{array}\right) \mathrm{d} s+\int_{0}^{t} \Sigma\left(T_{1}, T_{2}, s\right) \mathrm{d} N_{2}(s)
$$

for any $t \leq T_{1}$. Here, $N_{2}(t)=\left(B_{1}(\Theta(t)), B_{2}(\Theta(t))\right), t \geq 0$ where $B=\left(B_{1}, B_{2}\right)$ is $a \mathbb{R}^{2}$-valued standard Brownian motion and $\Sigma\left(T_{1}, T_{2}, s\right)$ is the non-negative $2 \times 2$ matrix-valued process with elements 


$$
\left(\Sigma\left(T_{1}, T_{2}, s\right)\right)_{i, j}^{2}=\left\langle\Psi(s) \mathcal{Q} \Psi^{*}(s) h_{T_{i}-s}, h_{T_{j}-s}\right\rangle
$$

for any $s, t \in \mathbb{R}_{+}, i, j=1,2$.

Proof This is along the same lines as the proof of Theorem 3.8.

We note that $\Sigma\left(T_{1}, T_{2}, s\right)_{i, i}$ will depend on $T_{i}$ only, for $i=1,2$. The dependency structure between $F\left(t, T_{1}\right)$ and $F\left(t, T_{2}\right)$ will be determined by $\Sigma\left(T_{1}, T_{2}, s\right)_{1,2}$, which we can interpret as the covariance in case of $L=W$ and $\Psi$ deterministic. This explicit representation of two forward contracts can be utilized in the analysis of calendar spread options, that is, options with payoff depending on $F\left(T, T_{1}\right)-F\left(T, T_{2}\right)$ at some exercise time $T \leq T_{1}$.

Example 3.10 Consider a simple example of a forward dynamics: Let $\Psi(s)=I$, the identity operator on $H_{w}$ and assume $W$ is a Wiener process with values in $U=H_{w}$. Furthermore, let $\beta=0$. Hence,

$$
f(t)=\mathcal{U}_{t} f_{0}+\int_{0}^{t} \mathcal{U}_{t-s} \mathrm{~d} W(s)
$$

Since $\delta_{y} \mathcal{U}_{s}=\delta_{y+s}$, we find

$$
F(t, T)=\delta_{T-t} f(t)=f_{0}(T)+\int_{0}^{t} \delta_{T-s} \mathrm{~d} W(s) .
$$

We have that $B_{T}(t):=\int_{0}^{t} \delta_{T-s} \mathrm{~d} W(s), t \in[0, T]$ and $T>0$ is a timeinhomogeneous Brownian motion. Its quadratic variation structure is given by $\mathrm{E}\left(B_{T}^{2}(t)\right)=\int_{0}^{t}\left(\mathcal{Q} h_{T-s}\right)(T-s) \mathrm{d} s$ and $\mathrm{E}\left(B_{T_{1}}(t) B_{T_{2}}(t)\right)=\int_{0}^{t}\left(\mathcal{Q} h_{T_{1}-s}\right)\left(T_{2}-s\right) \mathrm{d} s$. Note that the process $t \mapsto B_{t}(t)=\int_{0}^{t} \delta_{t-s} \mathrm{~d} W(s)$ becomes a Gaussian martingale, and

$$
S(t)=f_{0}(t)+B_{t}(t)
$$

is the spot price dynamics.

In view of Theorem 3.9, we would like to analyse in more detail the spatial correlation structure for random fields defined as stochastic processes with values in the Hilbert space $H_{w}$. For this purpose, we show first a technical Lemma, which reveals that two point evaluations on $H_{w}$ at close points are almost the same and hence cannot be orthogonal at all. In other words, the specific geometric structure of the space $H_{w}$ implies a strong connection between the different point evaluations.

Lemma 3.11 Recall the function $h_{x}: \mathbb{R}_{+} \rightarrow \mathbb{R}, z \mapsto 1+\int_{0}^{x \wedge z} \frac{1}{w(s)} d s$ for any $x \in \mathbb{R}_{+}$defined in Lemma 3.1. Then

$$
h: \mathbb{R}_{+} \rightarrow H_{w}, x \mapsto h_{x}
$$


is 0.5-Hölder continuous with constant 1 . Moreover,

$$
\left\|h_{y}-h_{x}\right\|_{w} \geq \sqrt{\frac{y-x}{w(y)}}
$$

for any $0 \leq x \leq y<\infty$.

Proof Let $0 \leq x \leq y<\infty$. Then

$$
\left\|h_{y}-h_{x}\right\|_{w}^{2}=\int_{x}^{y} \frac{1}{w(s)} \mathrm{d} s\left\{\begin{array}{l}
\leq y-x \\
\geq \frac{y-x}{w(y)}
\end{array}\right. \text { and }
$$

and hence $\left\|h_{y}-h_{x}\right\|_{w} \leq \sqrt{y-x}$ and $\left\|h_{y}-h_{x}\right\|_{w} \geq \sqrt{(y-x) / w(y)}$.

Note that for $0 \leq x \leq y<\infty$ we have $\left\langle h_{y}, h_{x}\right\rangle=\left\|h_{x}\right\|_{w}^{2}$, and therefore it also holds that

$$
\frac{\left\langle h_{y}, h_{x}\right\rangle}{\left\|h_{y}\right\|_{w}\left\|h_{x}\right\|_{w}}=\frac{\left\|h_{x}\right\|_{w}}{\left\|h_{y}\right\|_{w}} .
$$

Consider now a square integrable random variable $X$ with values in $H_{w}$ having mean zero and covariance operator $\mathcal{Q}$. The correlation between $X(x):=\delta_{x}(X)$ and $X(y)=\delta_{y}(X)$ is given as

$$
\rho: \mathbb{R}_{+}^{2} \rightarrow \mathbb{R},(x, y) \mapsto \begin{cases}\frac{\mathrm{E}\left[\delta_{x}(X) \delta_{y}(X)\right]}{\sqrt{\mathrm{E}\left[\delta_{x}(X)^{2}\right] \mathrm{E}\left[\delta_{y}(X)^{2}\right]}} & \text { if the denominator is non-zero, } \\ 1 & \text { otherwise. }\end{cases}
$$

The next Theorem shows, based on the Lemma above, that the correlation converges to one at a rate $\sqrt{|x-y|}$ as $x \rightarrow y$ :

Theorem 3.12 Let $X$ be a mean zero, square integrable and $H_{w}$-valued random variable with covariance operator $\mathcal{Q}$. For any $x \in \mathbb{R}_{+}$there is $\epsilon>0$ such that for $\rho$ defined in (11) it holds

$$
\rho(x, y) \geq 1-\frac{2\|\mathcal{Q}\|_{\text {op }}^{1 / 2} \sqrt{|x-y|}}{\left\|\mathcal{Q}^{1 / 2} h_{x}\right\|_{w}+\|\mathcal{Q}\|_{\text {op }}^{1 / 2} \sqrt{|x-y|}}
$$

for any $|x-y| \leq \epsilon$.

Proof Denote by $C:=\mathcal{Q}^{1 / 2}$ the positive semidefinite square root of $Q$ and let $x \in$ $\mathbb{R}_{+}$. If $\left\|\mathcal{Q}^{1 / 2} h_{x}\right\|_{w}=0$, then the claimed inequality is trivial. Thus, we assume that $\left\|\mathcal{Q}^{1 / 2} h_{x}\right\|_{w} \neq 0$.

Define

$$
\epsilon:=\frac{\left\|C h_{x}\right\|_{w}^{2}}{\|\mathcal{Q}\|_{\mathrm{op}}}
$$


and let $y \in \mathbb{R}_{+}$such that $|y-x|<\epsilon$. Then Lemma 3.11 yields

$$
\left\|C h_{y}\right\|_{w} \geq\left\|C h_{x}\right\|_{w}-\|C\|_{\mathrm{op}} \sqrt{|y-x|}>0
$$

and hence

$$
\rho(x, y)=\frac{\mathrm{E}\left[\delta_{x}(X) \delta_{y}(X)\right]}{\left\|C h_{x}\right\|_{w}\left\|C h_{y}\right\|_{w}} .
$$

We have

$$
\mathrm{E}\left(\delta_{x}(X) \delta_{y}(X)\right)=\left\langle\mathcal{Q} h_{x}, h_{y}\right\rangle
$$

by Lemma 3.1. Moreover, we have

$$
\mathrm{E}\left[\delta_{x}(X)^{2}\right]=\left\langle\mathcal{Q} h_{x}, h_{x}\right\rangle=\left\|C h_{x}\right\|_{w}^{2}
$$

and

$$
\begin{aligned}
\left\|C h_{y}\right\|_{w} & \leq\left\|C h_{x}\right\|_{w}+\|C\|_{\mathrm{op}}\left\|h_{y}-h_{x}\right\|_{w} \\
& \leq\left\|C h_{x}\right\|_{w}+\|C\|_{\mathrm{op}} \sqrt{|y-x|} .
\end{aligned}
$$

Thus, we get

$$
\begin{aligned}
\mathrm{E}\left[\delta_{x}(X) \delta_{y}(X)\right] & =\left\langle C h_{x}, C h_{y}\right\rangle \\
& \geq\left\langle C h_{x}, C h_{x}\right\rangle-\left|\left\langle C h_{x}, C\left(h_{y}-h_{x}\right)\right\rangle\right| \\
& \geq\left\|C h_{x}\right\|_{w}^{2}-\left\|C h_{x}\right\|_{w}\|C\|_{\mathrm{op}}\left\|h_{y}-h_{x}\right\|_{w} \\
& \geq\left\|C h_{x}\right\|_{w}\left(\left\|C h_{x}\right\|_{w}-\|C\|_{\mathrm{op}} \sqrt{|y-x|}\right),
\end{aligned}
$$

where the third inequality follows from the Cauchy-Schwarz inequality. We conclude

$$
\begin{aligned}
\rho(x, y) & \geq \frac{\left\|C h_{x}\right\|_{w}-\|C\|_{\mathrm{op}} \sqrt{|y-x|}}{\left\|C h_{x}\right\|_{w}+\|C\|_{\mathrm{op}} \sqrt{|y-x|}} \\
& =1-\frac{2\|\mathcal{Q}\|_{\mathrm{op}}^{1 / 2} \sqrt{|y-x|}}{\left\|\mathcal{Q}^{1 / 2} h_{x}\right\|_{w}+\|\mathcal{Q}\|_{\mathrm{op}}^{1 / 2} \sqrt{|y-x|}}
\end{aligned}
$$

because $\|\mathcal{Q}\|_{\text {op }}^{1 / 2}=\left\|\mathcal{Q}^{1 / 2}\right\|_{\text {op }}$ by Palmer [38, Theorem 3.4.21].

Observe, that there is no a priori upper bound for the correlation. Indeed, consider the random variable $X:=\left(h_{x}+h_{y}\right) A$ where $A$ is a standard normal random variable, $0 \leq x \leq y \leq \infty$. Then, $\rho(x, y)=1$.

Theorem 3.12 implies that the correlation among forwards with close maturities is high, which is very natural from a practical perspective as there is little economical 
difference of having a commodity delivered at different but nearby times. However, it is remarkable that the spatial geometry of the space $H_{w}$ is imposing an explicit lower bound for the correlation, which is stationary in distance between times to delivery and following a square-root function.

\subsection{Representing the Forwards by a Sum of Ornstein-Uhlenbeck Type Processes}

In commodity markets, a popular class of spot price models is given in terms of a finite sum of Ornstein-Uhlenbeck processes (see, e.g., Benth et al. [8] and the references therein). In the traditional set-up, one is modelling the logarithmic spot price dynamics by a finite sum of Ornstein-Uhlenbeck processes, each driven by a Lévy process. However, several papers also advocate to model the spot price dynamics directly by such a finite sum of Ornstein-Uhlenbeck processes (see, e.g., Benth et al. [10] and Garcia et al. [28] for the case of power). In a very simplistic setting, one starts out with a model

$$
S(t)=\Lambda(t)+X(t)
$$

where $\Lambda$ is some deterministic seasonality function, and $X$ follows an OrnsteinUhlenbeck process

$$
d X(t)=-\lambda X(t) \mathrm{d} t+\mathrm{d} N(t)
$$

for some $\mathbb{R}$-valued Lévy process $N$ and $\lambda>0$ a constant. One defines the forward price to be the conditional expectation of the spot price at delivery, given information at current time, where the expectation is taken under some pricing measure (see Benth et al. [8]). I.e.,

$$
F(t, T)=\mathrm{E}\left[S(T) \mid \mathcal{F}_{t}\right]
$$

for $t \leq T$. To avoid unnecessary technical details, we assume that the spot model is already stated under the pricing measure, and that the Lévy process $N$ is integrable. Then it is a straightforward task to derive that

$$
F(t, T)=\Lambda(T)+\frac{1}{\lambda} \mathrm{E}[N(1)]\left(1-e^{-\lambda(T-t)}\right)+e^{-\lambda(T-t)} X(t) .
$$

In other words, the forward price is linear in the Ornstein-Uhlenbeck process, with a coefficient which is exponentially decreasing in time to maturity $T-t$ at a rate given by the speed of mean reversion $\lambda$. If we consider a spot model being a sum of such Ornstein-Uhlenbeck processes, we find that the above expression for the forward price will generalise naturally to a sum of exponentially weighted Ornstein-Uhlenbeck processes.

We want now to analyse to what extent the opposite holds, that is, when can we represent our forward price dynamics $f(t)$ being a process with values in $H_{w}$ as a weighted series of Ornstein-Uhlenbeck processes? Of course, as long as we are letting 
the forward price be perturbed by an infinite-dimensional noise, we cannot expect a finite sum of Ornstein-Uhlenbeck processes (appropriately weighted) representing the stochastic evolution of the curve in $H_{w}$, but an infinite representation may be within reach. The main technical obstacle for such an infinite series representation is that the exponential functions do not constitute any orthogonal set in $H_{w}$. However, if the forward curve takes values in a convenient subspace of $H_{w}$ having exponential functions as a Riesz basis, it turns out that we can find a series representation in terms of Ornstein-Uhlenbeck processes. We present some auxiliary results to show this.

Recall that a Riesz basis is a sequence $\left(x_{n}\right)_{n \in \mathbb{N}}$ in a Hilbert space $H$ such that there is an orthonormal basis $\left(e_{n}\right)_{n \in \mathbb{N}}$ in $H$ and an invertible linear operator $\mathcal{T}$ such that $\mathcal{T} e_{n}=x_{n}$. For further equivalent statements, we refer the reader to Young [46, Theorem 1.9]. In the next Theorem, we find a Riesz basis consisting only of exponential functions for a 'large' subspace of $H_{w}$. This subspace will contain a natural copy of the first Sobolev space over $L^{2}([0, T], \mathbb{C})$. We formulate our results for the particular choice of weight functions $w: x \mapsto e^{\alpha x}$ for $\alpha>0$ in $H_{w}$ :

Theorem 3.13 Let $\lambda>0$, and fix $x_{0}>0$. Then there is a closed subspace $H_{w}^{x_{0}}$ such that the following statements hold.

(1) $H_{w}^{x_{0}}$ has a Riesz basis $\left(g_{n}\right)_{n \in \mathbb{Z}}$ such that $g_{0}(x)=1, x \in \mathbb{R}_{+}$and $g_{n}(x)=$ $\frac{1}{\lambda_{n} \sqrt{x_{0}}}\left(1-e^{\lambda_{n} x}\right), x \in \mathbb{R}_{+}, n \neq 1$ where $\lambda_{n}=\frac{2 \pi \text { in }}{x_{0}}-\lambda-\alpha / 2$.

(2) $\bar{g}_{n}=g_{-n}$ for any $n \in \mathbb{C}$.

(3) There is a continuous linear projection $\Pi_{x_{0}}: H_{w} \rightarrow H_{w}^{x_{0}}$ such that $\Pi_{x_{0}} g(x)=$ $g(x)$ for any $g \in H_{w}, x \in\left[0, x_{0}\right]$.

(4) $H_{w}^{x_{0}}$ is invariant under the semigroup $\left(\mathcal{U}_{t}\right)_{t \geq 0}$ defined in Theorem 3.4.

(5) If $\left(g_{n}^{*}\right)_{n \in \mathbb{Z}}$ is the corresponding biorthogonal system in the sense of Young [46, p. 28] and $\left(\mathcal{U}_{t}\right)_{t \geq 0}$ the semigroup defined in Theorem 3.4, then $\mathcal{U}_{t}^{*} g_{n}^{*}=e^{-\lambda_{n} t} g_{n}^{*}$ and $g_{0}^{*}=g_{0}$.

Proof Let $V$ be as in Lemma 4.22. Then Lemma 4.22 states that $\left(\tilde{g}_{n}\right)_{n \in \mathbb{Z}}$ is a Riesz basis of $V$ where

$$
\tilde{g}_{n}: \mathbb{R}_{+} \rightarrow \mathbb{C}, x \mapsto \frac{1}{\sqrt{x_{0}}} e^{\left(\frac{2 \pi i n}{x_{0}}-\lambda\right) x}, \quad n \in \mathbb{N} .
$$

Define $H_{w}^{x_{0}}:=\left\{f \in H_{w}: f^{\prime} \sqrt{w} \in V\right\}$ and

$$
\begin{gathered}
g_{n}(x):=\int_{0}^{x} \tilde{g}_{n}(y) e^{-y \alpha / 2} \mathrm{~d} y=\frac{1-e^{\lambda_{n} x}}{\lambda_{n} \sqrt{x_{0}}}, \quad x \in \mathbb{R}_{+} \cdot \\
\Psi: L^{2}\left(\mathbb{R}_{+}, \mathbb{C}\right) \rightarrow H_{w}, f \mapsto\left(x \mapsto \int_{0}^{x} f(y) e^{-y \alpha / 2} \mathrm{~d} y\right)
\end{gathered}
$$

is an isometric embedding and hence (1) and (2) follow. For the remainder of the proof, let $\left(g_{n}\right)_{n \in \mathbb{Z}}$ be as in (1). 
Let $F:=\left\{f \in H_{w}: f(x)=0, x \in\left[0, x_{0}\right]\right\}$. Then $F$ is a closed vector space complement to $H_{w}^{x_{0}}$ in $H_{w}$. Hence there is a continuous linear projection $\Pi: H_{w} \rightarrow$ $H_{w}^{x_{0}}$ with kernel $F$. Let $g \in H_{w}, x \in\left[0, x_{0}\right]$. Then $g-\Pi g$ is in the kernel of $\Pi$. Hence $g-\Pi g \in F$ which implies (3).

Let $\left(\mathcal{U}_{t}\right)_{t \geq 0}$ be the shift semigroup defined in Theorem 3.4. Then

$$
\mathcal{U}_{t} g_{n}=e^{-\lambda_{n} t} g_{n}+g_{n}(t) g_{0} \in H_{w}^{x_{0}},
$$

which shows (4).

Since $g_{0}$ is normed and orthogonal to $\left(g_{n}\right)_{n \neq 0}$, we have $g_{0}^{*}=g_{0}$. Young [46, Theorem 1.8] implies that $\left(g_{n}^{*}\right)_{n \in \mathbb{Z}}$ is a Riesz basis as well. Let $n \neq 0$. Then we have

$$
\begin{aligned}
\mathcal{U}_{t}^{*} g_{n}^{*} & =\sum_{k \in \mathbb{Z}}\left\langle\mathcal{U}_{t}^{*} g_{n}^{*}, g_{k}\right\rangle g_{k}^{*} \\
& =\sum_{k \in \mathbb{Z}}\left\langle g_{n}^{*}, \mathcal{U}_{t} g_{k}\right\rangle g_{k}^{*} \\
& =\sum_{k \in \mathbb{Z}}\left\langle g_{n}^{*}, e^{-\lambda_{k} t} g_{k}+g_{k}(t) g_{0}\right\rangle g_{k}^{*} \\
& =e^{-\lambda_{n} t} g_{n}^{*} .
\end{aligned}
$$

Hence, the proof is complete.

In view of Theorem 3.13, any stochastic process $X$ on $H_{w}$ can be mimicked by a stochastic process $Y$ on $H_{w}^{x_{0}}$, i.e., $Y(t, x)=X(t, x)$ for any $x \in\left[0, x_{0}\right]$. If we assume that the forwards price process $f(t)$ evolves in the space $H_{w}^{x_{0}}$, then it can be represented by a sum of Ornstein-Uhlenbeck type processes.

Theorem 3.14 Let $x_{0}>0$ and $H_{w}^{x_{0}}$ be as in Theorem 3.13. Assume that $f(t)$ is $H_{w}^{x_{0}}$-valued. Then there is a sequence $\left(M_{n}\right)_{n \in \mathbb{N}}$ of complex valued square integrable martingales such that

$$
f(t)=S(t)+2 \sum_{n=1}^{\infty} \operatorname{Re}\left(g_{n} \int_{0}^{t} e^{(s-t) \lambda_{n}}\left\{\left\langle g_{n}^{*}, \beta(s)\right\rangle \mathrm{d} s+\mathrm{d} M_{n}(s)\right\}\right), \quad t \in \mathbb{R}_{+} .
$$

where the sum converges almost surely in $H_{w}$ and $\left(g_{n}\right)_{n \in \mathbb{Z}}$ is the Riesz basis provided in Theorem 3.13.

Moreover, if $\Psi$ is deterministic, then $M_{n}$ is a process with independent increments. If $\Psi$ is deterministic and constant, then $M_{n}$ is a Lévy process. If $\Psi$ is deterministic and $L$ a Brownian motion, then $\left(M_{n}\right)_{n \geq 1}$ is a sequence of Gaussian processes.

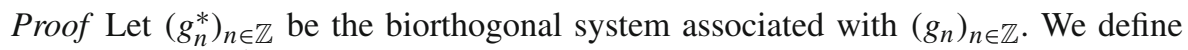
$M_{n}(t):=\left\langle g_{n}^{*}, \int_{0}^{t} \Psi_{s} d L(s)\right\rangle$ for any $n \in \mathbb{Z}$. Observe that $M_{n}, n \geq 1$ have the properties stated at the end of the theorem. We have 


$$
\begin{aligned}
f(t) & =\sum_{n \in \mathbb{Z}} g_{n}\left\langle f(t), g_{n}^{*}\right\rangle \\
& =\left\langle f(t), g_{0}^{*}\right\rangle+\sum_{n \in \mathbb{Z}, n \neq 0}\left(g_{n} \int_{0}^{t}\left\langle g_{n}^{*}, \mathcal{U}_{t-s} \beta(s) \mathrm{d} s\right\rangle+g_{n} \int_{0}^{t}\left\langle g_{n}^{*}, \mathcal{U}_{t-s} \Psi(s) \mathrm{d} L(s)\right\rangle\right)
\end{aligned}
$$

for any $t \in \mathbb{R}_{+}$. As $g_{0}^{*}=1$, we find $\left\langle f(t), g_{0}^{*}\right\rangle=S(t)$. Moreover, we have

$$
\begin{aligned}
\int_{0}^{t}\left\langle g_{n}^{*}, \mathcal{U}_{t-s} \Psi(s) \mathrm{d} L(s)\right\rangle & =\int_{0}^{t}\left\langle\mathcal{U}_{t-s}^{*} g_{n}^{*}, \Psi(s) \mathrm{d} L(s)\right\rangle \\
& =\int_{0}^{t} e^{(s-t) \lambda_{n}}\left\langle g_{n}^{*}, \Psi(s) \mathrm{d} L(s)\right\rangle \\
& =\int_{0}^{t} e^{(s-t) \lambda_{n}} \mathrm{~d} M_{n}(s) .
\end{aligned}
$$

Here we have applied Theorem 3.13, part (5) in the second equality. Similarly for the drift part $\beta$ we calculate,

$$
\begin{aligned}
\int_{0}^{t}\left\langle g_{n}^{*}, \mathcal{U}_{t-s} \beta(s) \mathrm{d} s\right\rangle & =\int_{0}^{t}\left\langle\mathcal{U}_{t-s}^{*} g_{n}^{*}, \beta(s) \mathrm{d} s\right\rangle \\
& =\int_{0}^{t} e^{(s-t) \lambda_{n}}\left\langle g_{n}^{*}, \beta(s) \mathrm{d} s\right\rangle \\
& =\int_{0}^{t} e^{(s-t) \lambda_{n}}\left\langle g_{n}^{*}, \beta(s)\right\rangle \mathrm{d} s
\end{aligned}
$$

for any $n \in \mathbb{Z}, n \neq 0, t \in \mathbb{R}_{+}$. Finally, observe that $g_{n} \int_{0}^{t} e^{(s-t) \lambda_{n}} \mathrm{~d} M_{n}(s)$ is the complex conjugate of $g_{-n} \int_{0}^{t} e^{(s-t) \lambda_{-n}} \mathrm{~d} M_{-n}(s)$ and hence their sum equals $2 \operatorname{Re}\left(g_{n} \int_{0}^{t} e^{(s-t) \lambda_{n}} \mathrm{~d} M_{n}(s)\right)$.

The Theorem tells us that the forward curves are indeed representable as an infinite series of (complex-valued) Ornstein-Uhlenbeck processes. We must restrict our attention to the space $H_{w}^{x_{0}}$, however, as we have from above, any element in $H_{w}$ can be projected to $H_{w}^{x_{0}}$, and the two curves will coincide on $\left[0, x_{0}\right]$. We may view $x_{0}$ as the maximal horizon of time-to-maturities of interest in the market. For example, in power markets, typically, contracts have deliveries for up to 4 years (this is the case in the Nordic market NordPool, or the German EEX market). One may model the forward curve in $H_{w}$, project it down to $H_{w}^{x_{0}}$, and then have the representation in 
terms of Ornstein-Uhlenbeck processes. For the times of deliveries in question, i.e., for $x \leq x_{0}$, we will have that the representation coincides with the dynamics of $f(t)$. Outside, for $x>x_{0}$, we do not know if this is true for the curve in $H_{w}$.

Let us give one final remark on the specific weight function $w$. The case when $w$ is not an exponential function is very delicate and requires an extensive generalisation of the analysis above. Since we do not want to deviate from our main topic too much, we decided not to include the more general cases here.

\subsection{Factorial Models and Covariance Representation}

As we recall from Peszat and Zabczyk [40], $L$ can be written as a sum of orthogonal and uncorrelated real-valued Lévy processes based on the spectral decomposition of the covariance operator $\mathcal{Q}$. This decomposition can be used to express the forward dynamics $f(t)$ in terms of a series representation. However, in many practical applications, it is difficult to find the spectral decomposition of $\mathcal{Q}$ explicitly. Moreover, for modelling purposes, it is sometimes more convenient to specify the directions in which the driving noise pushes the forward curve. For instance, one would like to specify the covariance by

$$
\mathcal{Q} g=\sum_{n=1}^{k}\left\langle g_{n}, g\right\rangle g_{n},
$$

where $g_{1}, \ldots, g_{k}$ is a finite set of functions in $H_{w}$. In an empirical context, one may view these functions as factor loadings for observed directions. Note that the representation of $\mathcal{Q}$ in terms of the finite set of functions $\left\{g_{n}\right\}_{n=1}^{k}$ yields that the noise $L$ can be viewed as a $k$-dimensional Lévy process. This can be generalised to infinite sums as long as the extra condition $\sum_{n \in \mathbb{N}}\left\|g_{n}\right\|_{w}^{2}<\infty$ is satisfied. This section focuses on such decompositions and summarises our main results in this regard.

Remark 3.15 Let $\left(L_{n}\right)_{n \in I}, I \subseteq \mathbb{N}$ be a family of uncorrelated, mean-zero, square integrable Lévy processes with variance 1 and $\left(y_{n}\right)_{n \in I}$ a family in a Hilbert space $H$ such that $\sum_{n \in I}\left|y_{n}\right|^{2}<\infty$. Then

$$
\sum_{j=1}^{N} L_{n}(t) y_{n}, \quad t \in \mathbb{R}_{+}
$$

converges in $L^{2}(\Omega, H)$ to some $H$-valued mean-zero, square integrable Lévy process $L$ with covariance

$$
\mathcal{Q} x=\sum_{n \in I}\left\langle y_{n}, x\right\rangle y_{n}, \quad x \in H .
$$

If the sequence is stochastically independent, then Peszat and Zabczyk [40, Corollary $3.12]$ yield that the convergence is $P$-a.s. 
Although we are working in the particular Hilbert space $H_{w}$, the following result holds for general Hilbert spaces $H$ and we formulate it in such a case:

Theorem 3.16 Let $L$ be a square integrable Lévy process with covariance $\mathcal{Q}$ and $m:=\mathrm{EL}(1)$. Let $\left(y_{n}\right)_{n \in I}$ be a Riesz basis of $H$ with $I=\mathbb{N}$ or let $\left(y_{n}\right)_{n \in I}$ be a finite linear independent set of elements in $H$. Assume that the covariance $\mathcal{Q}$ of $L$ is given by

$$
\mathcal{Q} x=\sum_{n \in I}\left\langle x, y_{n}\right\rangle y_{n} \quad x \in H,
$$

where

$$
\sum_{n \in I}\left\|y_{n}\right\|^{2}<\infty
$$

Then there is a family $\left(L_{n}\right)_{n \in I}$ of uncorrelated, mean-zero, square integrable and $\mathbb{R}$-valued Lévy processes with $\mathrm{EL}_{n}^{2}(1)=1$ such that

- $L_{n}$ is adapted to the filtration generated by $L$ for any $n \in \mathbb{N}$,

- $L(t)=t m+\sum_{n \in \mathbb{N}} L_{n}(t) y_{n}$ where the sum converges in $L^{2}(\Omega, H)$ uniformly in $t$ on compact intervals.

In particular, if $W=L$ is a Wiener process, then $W_{n}:=L_{n}$ defines a family of independent standard Brownian motions and $W(t)=\sum_{n \in \mathbb{N}} W_{n}(t) y_{n}$ where the convergence is $P$-a.s. for any $t \in \mathbb{R}_{+}$.

Proof We may assume that $m=0$ because we can work with $\widetilde{L}(t):=L(t)-t m$ instead.

For any $n \in I$ let $z_{n} \in H$ such that $\left\langle z_{n}, y_{k}\right\rangle=1_{\{n \neq k\}}$ for any $k \in I$. Define

$$
L_{n}(t):=\left\langle z_{n}, L(t)\right\rangle, \quad t \in \mathbb{R}_{+} .
$$

Let $\Pi$ be the orthonormal projection onto the closed subspace generated by $\left(y_{n}\right)_{n \in I}$. We have

$$
\Pi(L(t))=\sum_{n \in I} L_{n}(t) y_{n} \quad \text { surely in } H
$$

for any $t \in \mathbb{R}_{+}$. Moreover, we have

$$
\begin{aligned}
\mathrm{E}\left(L_{n}(t) L_{k}(t)\right) & =t\left\langle\mathcal{Q} z_{n}, z_{k}\right\rangle \\
& =t\left\langle y_{n}, z_{k}\right\rangle \\
& =t 1_{\{n=k\}}
\end{aligned}
$$

for any $n, k \in I$. Thus, $\left(L_{n}\right)_{n \in I}$ is a family of uncorrelated, mean-zero, square integrable and $\mathbb{R}$-valued Lévy processes with $\mathrm{E} L_{n}^{2}(1)=1$. Moreover, observe that $\mathcal{Q} \Pi=\mathcal{Q}$ and hence we have 


$$
\begin{aligned}
\mathrm{E}\langle\Pi(L(t))-L(t), x\rangle^{2} & =t\|\mathcal{Q}(\Pi x-x)\|^{2} \\
& =0
\end{aligned}
$$

for any $x \in H$.

For the remainder of this Sect. 3.4, we do assume the following standing assumption

$$
\Psi(t)=\sigma(t) \mathcal{T}
$$

where $\sigma$ is some $\mathbb{R}$-valued, locally bounded and adapted stochastic process and $\mathcal{T}$ is a linear operator from $U$ to $H_{w}$. Then $\mathcal{T} \mathcal{Q} \mathcal{T}^{*}$ is a positive semidefinite trace-class operator. Hence, $\mathcal{T} \mathcal{Q} \mathcal{T}^{*}$ has a unique positive semidefinite root $C$, which is a HilbertSchmidt operator. Note that this specification of $\Psi$ is a simple approach to include seasonality and stochastic volatility into the forward curve evolution, while $\mathcal{T}$ embeds our Lévy process into our curve space $H_{w}$. For example, Barndorff-Nielsen et al. [4] and Benth [7] find evidence for stochastic volatility in power and gas spot prices, resp. Seasonality of volatility in forward prices is discussed from an empirical point of view for the NordPool market in Benth and Koekebakker [11], Andresen et al. [1] and Frestad et al. [27], where their results may also indicate presence of stochastic volatility. We have the following series representation of $f(t)$ :

Theorem 3.17 Let $\left(g_{n}\right)_{n \in I}$ be elements of $H_{w}$, satisfying either

- $I \subseteq \mathbb{N}$ is finite and $\left(g_{n}\right)_{n \in I}$ is linear independent, or

- $I=\mathbb{N}$ and $\left(g_{n}\right)_{n \in I}$ are Riesz-basis of a closed subspace of $H_{w}$ with $\sum_{n \in I}\left\|g_{n}\right\|_{w}^{2}$ $<\infty$.

Moreover, assume that $\left(\mathcal{T} \mathcal{Q} \mathcal{T}^{*}\right) g=\sum_{n \in I}\left\langle g_{n}, g\right\rangle g_{n}$ for any $g \in H_{w}$. Then there is a family of mean-zero $\mathbb{R}$-valued uncorrelated Lévy processes $\left(L_{n}\right)_{n \in I}$ with $\mathrm{E}\left(L_{n}(1)\right)^{2}=$ 1 such that

$$
f(t)=\mathcal{U}_{t} f_{0}+\int_{0}^{t} \mathcal{U}_{t-s} \beta(s) \mathrm{d} s+\sum_{n \in I} \int_{0}^{t} \sigma(s) \mathcal{U}_{t-s} g_{n} \mathrm{~d} L_{n}(s)
$$

for any $t \geq 0$ where the sums converge in $L^{2}\left(\Omega, H_{w}\right)$. Moreover, if additionally $g_{n} \in \operatorname{dom}\left(\partial_{x}\right)$ for any $n \in I$, then

$$
\begin{aligned}
f(t)= & \mathcal{U}_{t} f_{0}+\int_{0}^{t} \mathcal{U}_{t-s} \beta(s) \mathrm{d} s \\
& +\sum_{n \in I}\left(\int_{0}^{t} \int_{0}^{s}\left(\mathcal{U}_{s-r} g_{n}^{\prime}\right) \sigma(r) \mathrm{d} L_{n}(r) \mathrm{d} s+g_{n} \int_{0}^{t} \sigma(s) \mathrm{d} L_{n}(s)\right)
\end{aligned}
$$

for any $t \in \mathbb{R}_{+}$where the sums converge in $L^{2}\left(\Omega, H_{w}\right)$. Moreover, under the additional assumption we have 


$$
\begin{aligned}
S(t)= & f_{0}(t)+\int_{0}^{t} \delta_{t-s} \beta(s) \mathrm{d} s+\sum_{n \in I} \int_{0}^{t} g_{n}(t-s) \sigma(s) \mathrm{d} L_{n}(s) \\
= & f_{0}(t)+\int_{0}^{t} \mathcal{U}_{t-s} \beta(s) \mathrm{d} s \\
& +\sum_{n \in I}\left(\int_{0}^{t} \int_{0}^{s} g_{n}^{\prime}(s-r) \sigma(r) \mathrm{d} L_{n}(r) \mathrm{d} s+g_{n}(0) \int_{0}^{t} \sigma(s) \mathrm{d} L_{n}(s)\right)
\end{aligned}
$$

for any $t \in \mathbb{R}_{+}$.

Proof The representations for $S$ simply follow from applying the continuous linear functional $\delta_{0}$ to the representations of $f$.

Theorem 3.16 applied to the Lévy process $\mathcal{T}(L(t))_{t \geq 0}$ whose covariance is $\mathcal{T} \mathcal{Q} \mathcal{T}^{*}$ yields a family of mean-zero $\mathbb{R}$-valued uncorrelated Lévy processes $\left(L_{n}\right)_{n \in I}$ with $\mathrm{E}\left(L_{n}^{2}(1)\right)=1$ such that

$$
\mathcal{T}(L(t))=\sum_{n \in I} g_{n} \mathrm{~d} L_{n}(t)
$$

Thus, we have

$$
\begin{aligned}
\int_{0}^{t} \mathcal{U}_{t-s} \Psi(s) \mathrm{d} L(s) & =\int_{0}^{t} \sum_{n \in I}\left(\mathcal{U}_{t-s} g_{n}\right) \sigma(s) \mathrm{d} L_{n}(s) \\
& =\sum_{n \in I} \int_{0}^{t}\left(\mathcal{U}_{t-s} g_{n}\right) \sigma(s) \mathrm{d} L_{n}(s)
\end{aligned}
$$

for any $t \geq 0$. This yields the first representation for $f$. Under the additional assumption, we have

$$
\int_{0}^{t} \mathcal{U}_{t-s} g_{n} \sigma(s) \mathrm{d} L_{n}(s)=\int_{0}^{t} \int_{0}^{s} \mathcal{U}_{s-r} g_{n}^{\prime} \sigma(r) \mathrm{d} L_{n}(r) d s+g_{n} \int_{0}^{t} \sigma(s) \mathrm{d} L_{n}(s),
$$

which finalises the proof.

Exponential curves are of course of particular interest, i.e., curves of the form $g_{n}(x)=\lambda_{n} e^{-\gamma_{n} x}$. In this case, one can calculate the integrals more explicitly and it turns out that the forward curve $f(t)$ corresponds to a sum of Lévy-driven OrnsteinUhlenbeck type processes:

Corollary 3.18 Let $I=\{1, \ldots, d\}, d \in \mathbb{N},\left(\gamma_{n}\right)_{n \in I}$ a family of pairwise different elements in $\mathbb{R}_{+}$and $\left(\lambda_{n}\right)_{n \in I}$ be a family in $\mathbb{R}, g_{n}(x):=\lambda_{n} e^{-\gamma_{n} x}$ for $n \in I, x \in \mathbb{R}_{+}$ and assume that 
- $g_{n} \in H_{w}$, and hence $g_{n}$ is in the domain of $\partial_{x}$, for any $n \in I$,

- $\mathcal{T} \mathcal{Q} \mathcal{T}^{*} g=\sum_{n \in I}\left\langle g_{n}, g\right\rangle g_{n}, \quad g \in H_{w}$ and

- $\beta$ has values in the vector space generated by $g_{n}, n \in I$.

Then

$$
f(t)=\mathcal{U}_{t} f_{0}+\sum_{n \in I} g_{n} X_{n}(t)
$$

where

$$
\mathrm{d} X_{n}(t)=\left(\mu_{n}(t)-\gamma_{n} X_{n}(t)\right) \mathrm{d} t+\sigma(t) \mathrm{d} L_{n}(t)
$$

for any $t \in \mathbb{R}_{+}$where $L_{n}$ is as in Theorem 3.17 and $\mu_{n}: \mathbb{R}_{+} \times \Omega \rightarrow \mathbb{R}$ is some predictable process. Moreover, we have

$$
S(t)=f_{0}(t)+\sum_{n \in I} Y_{n}(t)
$$

where $\mathrm{d} Y_{n}(t)=\left(\mu_{n}(t)-\gamma_{n} Y_{n}(t)\right) \mathrm{d} t+\lambda_{n} \sigma(t) \mathrm{d} L_{n}(t)$ for any $t \in \mathbb{R}_{+}$.

Proof Since $g_{n}^{\prime}=-\lambda_{n} g_{n} \in H_{w}$, we have $g_{n}$ is in the domain of $\partial_{x}$. Since $\left(\gamma_{n}\right)_{n \in I}$ are pairwise different, we have that $\left(g_{n}\right)_{n \in I}$ is linearly independent. Let $V \subseteq H_{w}$ be the subspace generated by $g_{n}, n \in I$ and $\left(g_{n}^{*}\right)_{n \in I}$ be the biorthogonal system of $\left(g_{n}\right)_{n \in I}$ in $V$. Define

$$
\mu_{n}(t):=\left\langle g_{n}^{*}, \beta(t)\right\rangle, \quad t \in \mathbb{R}_{+}
$$

Then we have $\beta(t)=\sum_{n \in I} g_{n} \mu_{n}(t), t \geq 0$. Furthermore,

$$
\mathcal{U}_{t-s} g_{n}(x)=\lambda_{n} e^{-\gamma_{n}(x+t-s)}=g_{n}(x) e^{-\gamma_{n}(t-s)},
$$

which yields, using Theorem 3.17,

$$
\begin{aligned}
f(t) & =\mathcal{U}_{t} f_{0}+\int_{0}^{t} \mathcal{U}_{t-s} \beta(s) \mathrm{d} s+\sum_{n \in I} \int_{0}^{t} \sigma(s) \mathcal{U}_{t-s} g_{n} \mathrm{~d} L_{n}(s) \\
& =\mathcal{U}_{t} f_{0}+\int_{0}^{t} \mathcal{U}_{t-s} \sum_{n \in I} g_{n} \mu_{n}(s) \mathrm{d} s+\sum_{n \in I} g_{n} \int_{0}^{t} \sigma(s) e^{-\gamma_{n}(t-s)} \mathrm{d} L_{n}(s) \\
& =\mathcal{U}_{t} f_{0}+\sum_{n \in I} g_{n}\left(\int_{0}^{t} e^{-\gamma_{n}(t-s)} \mu_{n}(s) \mathrm{d} s+\int_{0}^{t} \sigma(s) e^{-\gamma_{n}(t-s)} \mathrm{d} L_{n}(s)\right) \\
& =\mathcal{U}_{t} f_{0}+\sum_{n \in I} g_{n}\left(\int_{0}^{t} e^{-\gamma_{n}(t-s)}\left(\mu_{n}(s) \mathrm{d} s+\sigma(s) \mathrm{d} L_{n}(s)\right)\right)
\end{aligned}
$$


Define the real-valued process

$$
X_{n}(t)=\int_{0}^{t} e^{-\gamma_{n}(t-s)}\left(\mu_{n}(s) \mathrm{d} s+\sigma(s) \mathrm{d} L_{n}(s)\right),
$$

which, by integration-by-parts, is an Ornstein-Uhlenbeck process

$$
\mathrm{d} X_{n}(t)=\left(\mu_{n}(t)-\gamma_{n} X_{n}(t)\right) \mathrm{d} t+\sigma(t) \mathrm{d} L_{n}(t)
$$

The representation for $S$ follows simply from (7) by applying $\delta_{0}$ to the representation of $f$.

Note that the condition $g_{n} \in H_{w}$ is of course equivalent to

$$
\int_{0}^{\infty} w(x) e^{-2 \gamma_{n} x} \mathrm{~d} x<\infty
$$

In the typical case where $w$ is an exponential function $w(x)=\exp (\alpha x)$ with $\alpha>0$, this condition is satisfied if and only if the coefficients $\gamma_{n}$ are strictly bigger than $\alpha / 2$. One possibility to ensure the second condition is to define the driving noise $L(t):=\sum_{n=1}^{d} g_{n} B_{n}(t)$ where $B$ is some $\mathbb{R}^{d}$-valued standard Brownian motion. In that specific setup, we have

Corollary 3.19 Let $I=\{1, \ldots, d\}, d \in \mathbb{N}, \alpha>0, w(x):=e^{\alpha x}$ for any $x \in \mathbb{R}_{+}$, $\left(\gamma_{n}\right)_{n \in I}$ a family of pairwise different elements in $\mathbb{R}_{+}$with $\gamma_{n}>\alpha / 2$ and $\left(\lambda_{n}\right)_{n \in I}$ be a family in $\mathbb{R}, g_{n}(x):=\lambda_{n} e^{-\gamma_{n} x}$ for $n \in I, x \in \mathbb{R}_{+}$and assume that

- $L(t)=W(t):=\sum_{n=1}^{d} g_{n} B_{n}(t)$,

- $\mathcal{T}$ is the identity operator on $H_{w}$,

- the market $(F(t, T))_{0 \leq t \leq T}$ does not allow for arbitrage in the sense of Delbaen and Schachermayer [21, Main theorem 1.1]

where $B=\left(B_{1}, \ldots, B_{d}\right)$ is an $\mathbb{R}^{d}$-valued standard Brownian motion. Then

$$
f(t)=\mathcal{U}_{t} f_{0}+\sum_{n \in I} g_{n} X_{n}(t)
$$

where

$$
\mathrm{d} X_{n}(t)=\left(\mu_{n}(t)-\gamma_{n} X_{n}(t)\right) \mathrm{d} t+\sigma(t) \mathrm{d} B_{n}(t)
$$

and $\mu_{n}: \mathbb{R}_{+} \times \Omega \rightarrow \mathbb{R}$ is some predictable process. Moreover, we have

$$
S(t)=f_{0}(t)+\sum_{n \in I} Y_{n}(t)
$$

where $\mathrm{d} Y_{n}(t)=\left(\mu_{n}(t)-\gamma_{n} Y_{n}(t)\right) \mathrm{d} t+\lambda_{n} \sigma(t) \mathrm{d} B_{n}(t)$ for any $t \in \mathbb{R}_{+}$. 
Proof We start to calculate the covariance operator $Q$ of $W$. Let $V \subseteq H_{w}$ be the vector space generated by $g_{1}, \ldots, g_{d}$. Then for any $h, g \in H_{w}$, where $h$ orthogonal to $V$, we have $\langle W(1), g\rangle=0$ and hence

$$
\langle\mathcal{Q} h, g\rangle=\mathrm{E}(\langle W(1), h\rangle\langle W(1), g\rangle)=0 .
$$

Let $\left(g_{1}^{*}, \ldots, g_{d}^{*}\right)$ be the biorthogonal system for $\left(g_{1}, \ldots, g_{d}\right)$ in $V$. For $n, k \in I$ we have

$$
\left\langle W(1), g_{n}^{*}\right\rangle=B_{n}(1)
$$

and hence $\left\langle\mathcal{Q} g_{n}^{*}, g_{k}^{*}\right\rangle=1_{\{n=k\}}$. Consequently, $\mathcal{Q} g=\sum_{n=1}^{d}\left\langle g, g_{n}\right\rangle g_{n}$.

Now we show that $\beta$ takes values in the vector space $V \subseteq H_{w}$ generated by $g_{1}, \ldots, g_{d}$. The fundamental theorem of asset pricing by Delbaen and Schachermayer [21, Main theorem 1.1] yields an equivalent probability measure $Q$ such that $(F(t, T))_{0 \leq t \leq T}$ is a $\sigma$-martingale under $Q$ for any $T>0$. Since the $P$-dynamics are given by

$$
F(t, T)=\mathcal{U}_{T} f_{0}+\int_{0}^{t} \mathcal{U}_{T-t} \beta(s) \mathrm{d} s+\int_{0}^{t} \mathcal{U}_{T-s} \sigma(s) \mathrm{d} W(s),
$$

we know, by using Jacod and Shiryaev [34, Theorem III.3.24], that $W(t)=W^{Q}(t)+$ $\int_{0}^{t} \beta^{Q}(s) \mathrm{d} s$ where $W^{Q}$ is a $Q$-Brownian motion and $\beta^{Q}: \mathbb{R}_{+} \times \Omega \rightarrow V$ is some predictable process. Hence the $Q$-dynamics are given by

$$
F(t, T)=\mathcal{U}_{T} f_{0}+\int_{0}^{t} \beta_{Q}(s) \mathrm{d} s+\int_{0}^{t} \sigma(s) \mathcal{U}_{T-s} \mathrm{~d} W^{Q}(s),
$$

where $\beta_{Q}(t):=\beta(t)+\sigma(t) \mathcal{U}_{T-t} \beta(t), t \geq 0$ is some predictable process. However, since $F(t, T), t \in[0, T]$ is a $\sigma$-martingale under $Q$ we have $\beta_{Q}=0$. Thus, $\mathcal{U}_{T-t} \beta(t)=-\sigma(t) \mathcal{U}_{T-t} \beta(t) \in V$ for any $0 \leq t \leq T$.

Several remarks are in place in connection to the Corollary above. First of all, we recall that typical spot price models in commodity markets are given as sums of Ornstein-Uhlenbeck processes. Lucia and Schwartz [37] propose, among other models, a Brownian driven Ornstein-Uhlenbeck dynamics for the spot price of power in the Nordic electricity market NordPool. They suggest a one- or two-factor model, where in the latter case, the Ornstein-Uhlenbeck process degenerates to drifted Brownian motion. Benth et al. [10] propose a general class of models for power prices defined as a sum of Ornstein-Uhlenbeck processes with different speeds of mean reversion, driven by Lévy processes. Corollary 3.18 tells us that our specific choice of the volatility structure $\Psi$ in the forward curve dynamics implies such a spot dynamics. 
The representation of the forward curve in terms of a finite sum of OrnsteinUhlenbeck processes can also be seen as a special case of the theory of finitedimensional realisations of forward rate models in fixed-income theory. In the paper of Björk and Gombani [14], it is shown that the solution of the stochastic partial differential equation for forward rates can be represented as a linear combination of Ornstein-Uhlenbeck processes for special affine-like volatility term structures. Their result matches very well with Corollary 3.18 above. The theory of Björk and Gombani [14] has later been significantly generalised by Filipović and Teichmann [23,24], Ekeland and Taflin [22], and more recently by Tappe [44]. The theory of Tappe [44] has been modified and slightly extended to forward curve models in commodity markets by Benth and Lempa [13], where the authors apply it to optimal portfolio management.

A simulation scheme for the forward price dynamics based on theory for numerical solution of hyperbolic stochastic differential equations has been designed and analysed in Barth and Benth [6]. The method applies a decomposition of the covariance operator along with a finite element method.

\section{Operators on $H_{w}$}

In this Section, we provide an in-depth analysis of various classes of operators on the space $H_{w}$ which are relevant in connection with forward price modelling. In the dynamics of the forward price $f(t)$ in (6), we have operators present in the volatility, $\Psi$ and the covariance $\mathcal{Q}$ of the noise $L$. Positive trace class operators play an important role for square integrable Lévy processes, cf. Peszat and Zabczyk [40, Sects. 4.4 and 4.6]. These operators are squares of symmetric Hilbert-Schmidt operators, and we provide a complete characterisation of these. It turns out that Hilbert-Schmidt operators on $H_{w}$ are almost integral operators. Moreover, we analyse the particular cases of integral and multiplication operators, which are highly relevant for concrete modelling purposes in commodities and energy.

Throughout this Section, the operator $\mathcal{W}$ mapping $H_{w}$ into $L^{2}\left(\mathbb{R}_{+}\right)$defined by

$$
\mathcal{W} f=\sqrt{w} f^{\prime}
$$

will become useful. It holds that

$$
\left(\delta_{0}, \mathcal{W}\right): H_{w} \rightarrow \mathbb{R} \times L^{2}\left(\mathbb{R}_{+}\right), f \mapsto\left(f(0), \sqrt{w} f^{\prime}\right)
$$

is an isometric isomorphism of the Hilbert spaces $H_{w}$ and $\mathbb{R} \times L^{2}\left(\mathbb{R}_{+}\right)$.

\subsection{Integral Operators}

A useful class of operators are integral operators. Naturally, an integral operator on $H_{w}$ is defined as

$$
\mathcal{I} f(x)=\int_{0}^{\infty} r(x, y) f(y) \mathrm{d} y
$$


for elements $f \in H_{w}$. Obviously, in order to have $\mathcal{I} f \in H_{w}$ depends on the integral kernel function $r: \mathbb{R}_{+}^{2} \mapsto \mathbb{R}$. However, using the representation

$$
f(y)=f(0)+\int_{0}^{y} f^{\prime}(z) \mathrm{d} z
$$

we find

$$
\begin{aligned}
\mathcal{I} f(x) & =\int_{0}^{\infty} r(x, y) \mathrm{d} y f(0)+\int_{0}^{\infty} r(x, y) \int_{0}^{y} f^{\prime}(z) \mathrm{d} z \mathrm{~d} y \\
& =\int_{0}^{\infty} r(x, y) \mathrm{d} y f(0)+\int_{0}^{\infty} \int_{z}^{\infty} r(x, y) \mathrm{d} y f^{\prime}(z) \mathrm{d} z .
\end{aligned}
$$

The first term in this representation is a multiplication of a function given by the kernel $r$ with the evaluation of $f$ at zero. The second term is again an integral operator, but now of the form

$$
\mathcal{T} f(x)=\int_{0}^{\infty} q(x, y) f^{\prime}(y) \mathrm{d} y
$$

for some kernel function $q: \mathbb{R}_{+}^{2} \mapsto \mathbb{R}$. We study these in the sequel, and consider multiplication operators in Sect. 4.3.

Definition 4.1 Let $q: \mathbb{R}_{+}^{2} \rightarrow \mathbb{R}$ be Borel measurable. The integral operator on $H_{w}$ with kernel $q$ is defined on its $\operatorname{domain} \operatorname{dom}(\mathcal{T})$ for those functions $f \in H_{w}$ which satisfy

(1) $\int_{0}^{\infty}\left|q(x, y) f^{\prime}(y)\right| \mathrm{d} y<\infty$ for any $x \in \mathbb{R}_{+}$and

(2) $\left(x \mapsto \int_{0}^{\infty} q(x, y) f^{\prime}(y) \mathrm{d} y\right) \in H_{w}$

and it is given by

$$
\mathcal{T} f(x):=\int_{0}^{\infty} q(x, y) f^{\prime}(y) \mathrm{d} y, \quad f \in \operatorname{dom}(\mathcal{T}), x \in \mathbb{R}_{+} .
$$

For example, one can realise the noise field $L$ on $H_{w}$ and let its covariance operator $\mathcal{Q}$ be represented as an integral operator. This means that we define $\mathcal{Q}$ via a kernel function $q$ as in the definition above. This kernel function gives a functional description of the correlation between two neighbouring locations along the curve $L(t)$. Also, letting $\Psi$ in the dynamics of $f$ in (5) be an integral operator, which enables us to mix the noise $L$ with a kernel function $q$, which in a sense is scaling the various noise sources for different locations to make up the noise in one. This is a natural generalisation of the 
case of finite-dimensional noise, where one is typically having a volatility which is a linear combination of the various components in the noise vector.

In order to understand integral operators, we proceed in the following way.

Remark 4.2 If $\mathcal{T}$ is an integral operator with kernel $q$, then it is everywhere defined, i.e., its domain equals $H_{w}$ if and only if

$\left(1^{\prime}\right) q(x, \cdot) / \sqrt{w} \in L^{2}\left(\mathbb{R}_{+}\right)$for any $x \in \mathbb{R}_{+}$and

(2') $\left(x \mapsto \int_{0}^{\infty} q(x, y) f^{\prime}(y) \mathrm{d} y\right) \in H_{w}$ for any $f \in H_{w}$.

The condition $\left(1^{\prime}\right)$ ensures that $f_{q}(x):=\int_{0}^{\infty} q(x, y) f^{\prime}(y) \mathrm{d} y, x \in \mathbb{R}_{+}$defines a measurable function. While condition $\left(2^{\prime}\right)$ ensures that $f_{q}$ is actually an element of $H_{w}$.

Sometimes an integral operator $\mathcal{T}$ with kernel $q$ can be extended to those functions $f \in H_{w}$ where the integral $\int_{0}^{\infty} q(x, y) f^{\prime}(y) \mathrm{d} y$ makes sense for any $x \geq 0$. However, we cannot expect that the resulting functions are members of $H_{w}$ or even continuous but as we show next they are measurable. This technical extension is needed later.

Lemma 4.3 Let $\mathcal{T}$ be an integral operator with kernel $q$ and $f \in H_{w}$ such that

$$
\int_{0}^{\infty}\left|q(x, y) f^{\prime}(y)\right| \mathrm{d} y<\infty
$$

for any $x \in \mathbb{R}_{+}$. Define

$$
f_{q}(x):=\int_{0}^{\infty} q(x, y) f^{\prime}(y) \mathrm{d} y
$$

for any $x \in \mathbb{R}_{+}$. Then $f_{q}$ is a measurable function.

Proof Let $r_{n}: \mathbb{R}_{+}^{2} \rightarrow \mathbb{R}$ such that $r_{n}$ is elementary $r_{n} \rightarrow q$ pointwise and $\left|r_{n}(x, y)\right| \leq 2|q(x, y)|$. Let $g_{n}: \mathbb{R}_{+} \rightarrow \mathbb{R}$ such that $g_{n}$ is elementary, $g_{n} \rightarrow f^{\prime}$ pointwise Lesbesgue-a.e. and $\left|g_{n}(x)\right| \leq 2\left|f^{\prime}(x)\right|$. Then $r_{n} g_{n} \rightarrow q f^{\prime}$ and $\left|r_{n} g_{n}\right| \leq 4\left|q f^{\prime}\right|$. Thus, the dominated convergence theorem yields

$$
h(x):=\int_{0}^{\infty} q(x, y) f^{\prime}(y) \mathrm{d} y=\lim _{n \rightarrow \infty} \int_{0}^{\infty} r_{n}(x, y) g_{n}(y) \mathrm{d} y .
$$

However, $h_{n}: \mathbb{R}_{+} \rightarrow \mathbb{R}, x \mapsto \int_{0}^{\infty} r_{n}(x, y) g_{n}(y) \mathrm{d} y$ is elementary and hence measurable. Thus, $h$ is the pointwise limit of measurable functions and hence measurable.

Recall, that an operator $\mathcal{T}$ is defined to be closed if for any sequence $\left(f_{n}\right)_{n \in \mathbb{N}}$ in the domain of $\mathcal{T}$ such that $f_{n}$ converges to some $f \in H_{w}$ and $\mathcal{T} f_{n}$ converges to some $g \in H_{w}$ we have $f$ is in the domain of $\mathcal{T}$ and $\mathcal{T} f=g$. Typically, on $L^{2}\left(\mathbb{R}_{+}\right)$integral 
operators, which are exactly defined for those functions where the integral expression yields an $L^{2}$-function, are not closed!, cf. Grafakos [29, Theorem I.1]. However, an integral operator $\mathcal{T}$ on $H_{w}$ satisfying condition $\left(1^{\prime}\right)$ is closed as we now show.

Lemma 4.4 Let $\mathcal{T}$ be an integral operator with kernel $q$. Assume that $\left(1^{\prime}\right)$ in Remark 4.2 is satisfied, i.e., $q(x, \cdot) / \sqrt{w} \in L^{2}\left(\mathbb{R}_{+}\right)$for any $x \in \mathbb{R}_{+}$. Then $\mathcal{T}$ is a closed linear operator.

Proof Linearity of $\mathcal{T}$ is obvious. Define $b(x, y):=q(x, y) / \sqrt{w(y)}$ for any $x, y \in \mathbb{R}_{+}$. Then $b(x, \cdot) \in L^{2}\left(\mathbb{R}_{+}\right)$for any $x \in \mathbb{R}_{+}$by assumption. Let $\left(f_{n}\right)_{n \in \mathbb{N}}$ be a sequence of elements in the domain of $\mathcal{T}$ which converges in $H_{w}$ to some $f$ such that $\mathcal{T} f_{n}$ converges to some $g$ in $H_{w}$. Recall the operator $\mathcal{W}$ defined in (15), which is a continuous linear operator. Thus, $\mathcal{W} f_{n} \rightarrow \mathcal{W} f$ in $L^{2}$. Let $x \in \mathbb{R}_{+}$. We have

$$
q(x, \cdot) f_{n}^{\prime}=b(x, \cdot) \mathcal{W} f_{n} \rightarrow b(x, \cdot) \mathcal{W} f=q(x, \cdot) f^{\prime},
$$

where the convergence is in $L^{1}\left(\mathbb{R}_{+}\right)$. Hence

$$
\begin{aligned}
\mathcal{T} f_{n}(x) & =\int_{0}^{\infty} q(x, y) f_{n}^{\prime}(y) \mathrm{d} y \\
& \rightarrow \int_{0}^{\infty} q(x, y) f^{\prime}(y) \mathrm{d} y \\
& =: h(x) .
\end{aligned}
$$

Lemma 3.1 yields that $\mathcal{T} f_{n}(x) \rightarrow g(x)$. Thus, $h=g$. Consequently, $h \in H_{w}$ which implies that $f \in \operatorname{dom}(\mathcal{T})$ and $g=\mathcal{T} f$.

The following corollary shows that an integral operator is continuous if and only if it is everywhere defined. This is a huge improvement compared to the statement in Remark 4.2. However, boundedness conditions for integral operators which are not everywhere defined are much more involved.

Corollary 4.5 Let $\mathcal{T}$ be an integral operator with kernel $q . \mathcal{T}$ is continuous if and only if the following two statements are satisfied.

(1) $q(x, \cdot) / \sqrt{w} \in L^{2}\left(\mathbb{R}_{+}\right)$for any $x \in \mathbb{R}_{+}$,

(2) $\left(x \mapsto \int_{0}^{\infty} q(x, y) f^{\prime}(y) \mathrm{d} y\right) \in H_{w}$ for any $f \in H_{w}$.

Proof Assume that the two conditions are satisfied. Then $\mathcal{T}$ is everywhere defined by definition. Lemma 4.4 yields that $\mathcal{T}$ is a closed operator. The closed graph theorem implies that $\mathcal{T}$ is a continuous linear operator.

Now assume that $\mathcal{T}$ is a continuous linear operator. Let $f \in H_{w}$ and $x \in \mathbb{R}_{+}$. Then $f$ is in the domain of $\mathcal{T}$ and consequently it satisfies condition (2) by definition and

$$
\left.\int_{0}^{\infty} \mid q(x, y) / w(y)\left(w f^{\prime}\right)(y)\right)\left|\mathrm{d} y=\int_{0}^{\infty}\right| q(x, y) f^{\prime}(y) \mid \mathrm{d} y<\infty .
$$


Since this is true for any $f \in H_{w}$ we conclude that $y \mapsto q(x, y) / w(y) \in L^{2}\left(\mathbb{R}_{+}\right)$. This proves the Corollary.

Let us consider an example which constitute an important case for power markets. In our definition of the forward curve dynamics $f(t)$, see (5), we model the forward price of contracts with a fixed time to delivery $x \in \mathbb{R}_{+}$. In the power market, one is trading in contracts delivering over a given period of time, and these contracts can be viewed as holding a portfolio of forwards delivering at each fixed time instant in the delivery period (see Benth and Koekebakker [11]). If we denote by $F^{\tau}(t, x)$ the forward price at time $t$ of a contract with time to delivery $x \in \mathbb{R}_{+}$and length of delivery $\tau \in \mathbb{R}_{+}$, we find that

$$
F^{\tau}(t, x)=\frac{1}{\tau} \int_{x}^{x+\tau} f(t, y) \mathrm{d} y .
$$

The forward price $F^{\tau}(t, x)$ is the average of the function $y \mapsto f(t, y)$ over the delivery period $[x, x+\tau]$ by market convention. From the representation

$$
f(t, y)=f(t, x)+\int_{x}^{y} \frac{\partial f}{\partial x}(t, z) \mathrm{d} z
$$

for $y \geq x$, we find from Fubini's theorem

$$
\begin{aligned}
F^{\tau}(t, x) & =\frac{1}{\tau} \int_{x}^{x+\tau}\left(f(t, x)+\int_{x}^{y} \frac{\partial f}{\partial x}(t, z) \mathrm{d} z\right) \mathrm{d} y \\
& =f(t, x)+\frac{1}{\tau} \int_{x}^{x+\tau} \int_{z}^{x+\tau} \mathrm{d} y \frac{\partial f}{\partial x}(t, z) \mathrm{d} z \\
& =f(t, x)+\int_{0}^{\infty} q^{\tau}(x, y) \frac{\partial f}{\partial x}(t, y) \mathrm{d} y
\end{aligned}
$$

with

$$
q^{\tau}(x, y)=\frac{1}{\tau}(x+\tau-y) \mathbf{1}_{[x, x+\tau]}(y) .
$$

Denote by $\mathcal{T}^{\tau}$ the integral operator with kernel $q^{\tau}$. We observe that the function $y \mapsto q^{\tau}(x, y)$ has support on $[x, x+\tau]$ for each given $x \in \mathbb{R}_{+}$, and hence Condition (1) in Corollary 4.5 is trivially satisfied since $w$ is a continuously increasing function with $w(0)=1$. Moreover, since 


$$
\left(\mathcal{T}^{\tau} f\right)^{\prime}(x)=\frac{1}{\tau} \frac{\mathrm{d}}{\mathrm{d} x} \int_{x}^{x+\tau}(x+\tau-y) f^{\prime}(y) \mathrm{d} y=\frac{f(x+\tau)-f(x)}{\tau}-f^{\prime}(x)
$$

it holds that

$$
\begin{aligned}
\int_{0}^{\infty} w(x)\left(\left(\mathcal{T}^{\tau} f\right)^{\prime}(x)+f^{\prime}(x)\right)^{2} \mathrm{~d} x & \leq \frac{1}{\tau} \int_{0}^{\infty} w(x) \int_{x}^{x+\tau}\left(f^{\prime}(y)\right)^{2} \frac{\mathrm{d} y}{\tau} \mathrm{d} x \\
& \leq \frac{1}{\tau} \int_{0}^{\infty} \int_{x}^{x+\tau} w(y)\left(f^{\prime}(y)\right)^{2} \frac{\mathrm{d} y}{\tau} \mathrm{d} x \\
& \leq \frac{1}{\tau} \int_{0}^{\infty} w(y)\left(f^{\prime}(y)\right)^{2} \mathrm{~d} y<\infty,
\end{aligned}
$$

for every $f \in H_{w}$, where we used respectively Jensen's inequality, that $w$ is increasing and Tonelli's theorem. Hence, by the triangle inequality we find,

$$
\left\|\mathcal{T}^{\tau} f\right\|_{w} \leq\left\|\mathcal{T}^{\tau} f+f\right\|_{w}+\|f\|_{w}<\infty
$$

This shows that Condition (2) of Corollary 4.5 is satisfied, which implies that $\mathcal{T}^{\tau}$ is a continuous integral operator on $H_{w}$. Moreover, it follows that $F^{\tau}(t) \in H_{w}$ for any forward curve $f(t) \in H_{w}$. Hence, we conclude that the power forward price dynamics for given length of delivery $\tau$ can be realised in $H_{w}$ as an application of a continuous integral operator along with the identity map on forward curves realised in $H_{w}$ :

$$
F^{\tau}(t)=\left(\operatorname{Id}+\mathcal{T}^{\tau}\right)(f(t))
$$

We note here that $\tau$, the length of delivery, is viewed as a parameter. One may in fact introduce this as a second variable, and introduce function spaces on $\mathbb{R}_{+}^{2}$ in a similar fashion as $H_{w}$. We leave this for forwards studies.

We continue with the general analysis of integral operators on $H_{w}$. In order to show that an integral operator defined by a function $q$ is bounded it is useful to consider Schur's lemma (see Grafakos [29, Appendix I.1]). Remark that this Lemma is sometimes known as Schur's test. However, we first have to identify integral operators on $H_{w}$ with integral operators on $L^{2}\left(\mathbb{R}_{+}\right)$.

Theorem 4.6 Let $\mathcal{T}$ be an integral operator on $H_{w}$ with kernel $q$. Assume that $q$ has an absolute derivative with respect to its first component, define

$$
b(x, y):=\partial_{1} q(x, y) \sqrt{w(x) / w(y)}
$$

and assume that 
(1) $q(0, \cdot) / \sqrt{w} \in L^{2}\left(\mathbb{R}_{+}\right)$,

(2) $\sup _{x \in \mathbb{R}} \int_{0}^{\infty}|b(x, y)| \mathrm{d} y=: A<\infty$ and

(3) $\sup _{y \in \mathbb{R}} \int_{0}^{\infty}|b(x, y)| \mathrm{d} x=: B<\infty$.

Then $\mathcal{T}$ is densely defined and bounded by

$$
c:=\left(\int_{0}^{\infty} \frac{q^{2}(0, y)}{w(y)} \mathrm{d} y+A B\right)^{1 / 2}
$$

Consequently, $\mathcal{T}$ can be uniquely extended to a continuous linear operator. Moreover, $b$ is locally integrable in its second variable and hence we can define the function

$$
q^{*}(y, x):=\int_{0}^{y} \sqrt{\frac{w(x)}{w(z)}} b(x, z) \mathrm{d} z .
$$

The dual of $\mathcal{T}$ is a continuous linear operator, bounded by $c$ and

$$
\mathcal{T}^{*} g(y)=\left(\int_{0}^{y} \frac{q(0, z)}{w(z)} \mathrm{d} z\right) g(0)+\int_{0}^{\infty} q^{*}(y, x) g^{\prime}(x) \mathrm{d} x,
$$

for $g \in H_{w}, y \in \mathbb{R}_{+}$such that $w g^{\prime} \in L^{1}\left(\mathbb{R}_{+}\right) \cap L^{\infty}\left(\mathbb{R}_{+}\right)$.

Moreover, if additionally

$$
\left(1^{\prime}\right) \quad q(x, \cdot) / \sqrt{w} \in L^{2}\left(\mathbb{R}_{+}\right)
$$

for any $x \in \mathbb{R}_{+}$, then $\mathcal{T}$ is a continuous linear operator. If $q^{*}(y, \cdot) / \sqrt{w} \in L^{2}\left(\mathbb{R}_{+}\right)$ for any $y \in \mathbb{R}_{+}$, then the representation for $\mathcal{T}^{*}$ extends to all functions in $H_{w}$.

Proof Schur's lemma, cf. Grafakos [29, Appendix I.1], yields that

$$
\widetilde{\mathcal{R}} f(x):=\int_{0}^{\infty} b(x, y) f(y) \mathrm{d} y, \quad f \in D(\widetilde{\mathcal{R}}), x \in \mathbb{R}_{+}
$$

is an operator on $L^{2}\left(\mathbb{R}_{+}\right)$with bound $(A B)^{1 / 2}$, whose domain includes $L^{1}\left(\mathbb{R}_{+}\right) \cap$ $L^{\infty}\left(\mathbb{R}_{+}\right)$. Since

$$
\partial_{1} q(x, y)=\sqrt{w(y) / w(x)} b(x, y),
$$

we conclude from Condition (2) and from local boundedness of $w$ that $\partial_{1} q$ is locally integrable. 
Let

$$
\widetilde{\mathcal{W}}: L^{2}\left(\mathbb{R}_{+}\right) \rightarrow H_{w}, g \mapsto \int_{0}^{x}(w(z))^{-1 / 2} g(z) \mathrm{d} z
$$

Then $\tilde{\mathcal{W}}$ is an isometry and $\mathcal{W} \widetilde{\mathcal{W}}$ is the identity on $L^{2}\left(\mathbb{R}_{+}\right)$where $\mathcal{W}$ is defined in (15). Thus, $\mathcal{R}:=\widetilde{\mathcal{W}} \widetilde{\mathcal{R}} \mathcal{W}$ is a densely defined operator with bound $(A B)^{1 / 2}$. Let $f \in H_{w}$ such that $f^{\prime}$ is continuous and has compact support $C$. Then $\mathcal{W} f \in \operatorname{dom}(\widetilde{\mathcal{R}})$ and hence $f \in \operatorname{dom}(\mathcal{R})$. Thus,

$$
\begin{aligned}
\mathcal{R} f(x) & =\int_{0}^{x}(w(z))^{-1 / 2} \int_{0}^{\infty} b(z, y) \sqrt{w(y)} 1_{C}(y) f^{\prime}(y) \mathrm{d} y \mathrm{~d} z \\
& =\int_{0}^{x} \int_{C} \partial_{1} q(z, y) f^{\prime}(y) \mathrm{d} y \mathrm{~d} z \\
& =\int_{C} \int_{0}^{x} \partial_{1} q(z, y) \mathrm{d} z f^{\prime}(y) \mathrm{d} y \\
& =\int_{C}(q(x, y)-q(0, y)) f^{\prime}(y) \mathrm{d} y,
\end{aligned}
$$

where we used Fubini's theorem for the second last equation. Condition (1) implies that

$$
\int_{0}^{\infty}\left|q(0, y) f^{\prime}(y)\right| \mathrm{d} y<\infty
$$

and hence we have $\mathcal{R} f(x)+\mathcal{T} f(0)=\mathcal{T} f(x), x \in \mathbb{R}_{+}$. The vector space

$$
V:=\left\{f \in H_{w}: \mathcal{W} f \in L^{1}\left(\mathbb{R}_{+}\right) \cap L^{\infty}\left(\mathbb{R}_{+}\right)\right\}
$$

is dense in $H_{w}$ because (16) states that $\left(\delta_{0}, \mathcal{W}\right)$ is an isometry,

$$
\left(\delta_{0}, \mathcal{W}\right)(V)=\mathbb{R} \times\left(L^{1}\left(\mathbb{R}_{+}\right) \cap L^{\infty}\left(\mathbb{R}_{+}\right)\right)
$$

and $L^{1}\left(\mathbb{R}_{+}\right) \cap L^{\infty}\left(\mathbb{R}_{+}\right)$is dense in $L^{2}\left(\mathbb{R}_{+}\right)$. Since $V$ is contained in the domain of $\mathcal{T}$, we have that $\mathcal{T}$ is densely defined. Now let $f \in \operatorname{dom}(\mathcal{T})$ with $\|f\| \leq 1$. Then the Pythagorean theorem, the Cauchy-Schwarz inequality and the bound for $\mathcal{R}$ imply 


$$
\|\mathcal{T} f\|^{2}=\|(x \mapsto \mathcal{T} f(0))\|^{2}+\|\mathcal{R} f\|^{2} \leq \int_{0}^{\infty} \frac{q^{2}(0, y)}{w(y)} \mathrm{d} y+A B
$$

Observe, that $q^{*}$ also satisfies conditions (1)-(3). Hence the integral operator $\mathcal{R}^{*}$ with kernel $q^{*}$ is densely defined and bounded by $(A B)^{1 / 2}$. Moreover, analogue computations to those above show that

$$
\mathcal{W} \mathcal{R}^{*} \widetilde{\mathcal{W}} g(x)=\int_{0}^{\infty} b(y, x) g(y) \mathrm{d} y
$$

for bounded compactly supported functions $g$, and hence $\mathcal{W} \mathcal{R}^{*} \widetilde{\mathcal{W}}$ is the dual of $\widetilde{\mathcal{R}}$. Consequently, $\mathcal{R}^{*}$ is the dual operator of $\mathcal{R}$. Now it is easy to deduce the dual operator of $\mathcal{T}$.

Lemma 4.4 states that the additional assumption implies that $\mathcal{T}$ is closed. However, a closed, bounded and densely defined operator is everywhere defined and continuous.

Remark 4.7 In the special case that $q(x, y)=q(0, y)$, we see that the dual of $\mathcal{T}$ is an operator which takes the initial value of the function and multiplies it with

$$
h(y):=\int_{0}^{y} \frac{q(0, z)}{w(z)} \mathrm{d} z
$$

Hence, one might write $\mathcal{T}^{*}=\mathcal{M}_{h} \delta_{0}$, where $\mathcal{M}_{h}$ denotes the multiplication operator by the function $h$. We will return to a more detailed study of general multiplication operators in Sect. 4.3.

A prominent class of integral operators is convolution type operators, i.e., operators of the form

$$
\mathcal{T} f(x)=\int_{0}^{\infty} k(x-y) f^{\prime}(y) \mathrm{d} y
$$

or, in other words, an integral operator with kernel $q(x, y):=k(x-y)$. The next Corollary restates the conditions of Theorem 4.6 for such convolution type operators. However, for simplicity, we will only do this in the case that the weight function $w$ in the space $H_{w}$ is an exponential function.

Corollary 4.8 Let $k: \mathbb{R} \rightarrow \mathbb{R}_{+}$be a measurable function and $\mathcal{T}$ be an integral operator on $H_{w}$ with kernel $q: \mathbb{R}_{+}^{2} \rightarrow \mathbb{R},(x, y) \mapsto k(x-y)$. Assume that

(1) $w(x)=e^{\alpha x}$ for some $\alpha>0$ and any $x \in \mathbb{R}_{+}$,

(2) $\int_{0}^{\infty}|k(x-y)|^{2} e^{-\alpha y} \mathrm{~d} y<\infty$ for any $x \in \mathbb{R}_{+}$, 
(3) $k$ has an absolutely continuous derivative and

(4) $\int_{-\infty}^{\infty}\left|k^{\prime}(s)\right| e^{\alpha s / 2} \mathrm{~d} s<\infty$.

Then $\mathcal{T}$ is a continuous linear operator.

Proof Define

$$
b(x, y):=\partial_{1} q(x, y) \sqrt{w(x) / w(y)}, \quad x, y \in \mathbb{R}_{+} .
$$

Then $b(x, y)=k^{\prime}(x-y) e^{1 / 2 \alpha(x-y)}$ for any $x, y \in \mathbb{R}_{+}$. Let $x \in \mathbb{R}_{+}$, then

$$
\int_{0}^{\infty}|b(x, y)| \mathrm{d} y=\int_{-\infty}^{x}\left|k^{\prime}(s)\right| e^{1 / 2 \alpha s} \mathrm{~d} s
$$

and hence

$$
\sup _{x \in \mathbb{R}_{+}} \int_{0}^{\infty}|b(x, y)| \mathrm{d} y=\int_{-\infty}^{\infty}\left|k^{\prime}(s)\right| e^{1 / 2 \alpha s} \mathrm{~d} s .
$$

Along the same lines we get

$$
\sup _{y \in \mathbb{R}_{+}} \int_{0}^{\infty}|b(x, y)| \mathrm{d} x=\int_{-\infty}^{\infty}\left|k^{\prime}(s)\right| e^{1 / 2 \alpha s} \mathrm{~d} s .
$$

We have

$$
\int_{0}^{\infty}|q(x, y) / \sqrt{w(y)}|^{2} \mathrm{~d} y=\int_{0}^{\infty}|k(x-y)|^{2} e^{-\alpha y} \mathrm{~d} y
$$

for any $x \in \mathbb{R}_{+}$. The claim follows from Theorem 4.6.

Let us end this Subsection on integral operators by coming back to the spot dynamics as presented in Theorem 3.8. Recall that the spot volatility is given by

$$
\sigma^{2}(t, s)=\left\langle\Psi(s) \mathcal{Q} \Psi^{*}(s) h_{t-s}, h_{t-s}\right\rangle
$$

Here, $\mathcal{Q}$ is the covariance operator of the driving noise of the forward dynamics $f(t)$ and $\Psi$ is the volatility operator in its dynamics. If we assume now that $\Psi(s)=\widetilde{\sigma}(s) \mathcal{T}$, for an integral operator on $H_{w}$

$$
\mathcal{T} g(x)=\int_{0}^{\infty} \psi(x, y) g^{\prime}(y) \mathrm{d} y \text {. }
$$


Observe that $\tilde{\sigma}$ is a predictable $\mathbb{R}$-valued stochastic process. We also assume $\mathcal{Q}$ to be an integral operator on $H_{w}$, cf. Theorem 4.11,

$$
\mathcal{Q} g(x)=\int_{0}^{\infty} q(x, y) g^{\prime}(y) \mathrm{d} y .
$$

Under the assumptions above, we can compute $\sigma^{2}(t, s)$ and find that

$$
\sigma^{2}(t, s)=\tilde{\sigma}^{2}(s) \int_{0}^{\infty} \int_{0}^{\infty} \frac{1}{w(u)} \psi(t-s, z) q_{1}(z, u) \psi(t-s, u) \mathrm{d} u \mathrm{~d} z,
$$

where $q_{1}$ is the partial derivative of $q$ with respect to its first argument. Thus, $\sigma^{2}(t, s)$ will be of the form

$$
\sigma(t, s)=\tilde{\sigma}(s) \gamma(t-s)
$$

which gives an LSS process (see Barndorff-Nielsen et al. [4]). We see that the deterministic kernel function $\gamma(t-s)$ will be an integral in terms of the kernels from both the volatility operator $\Psi$ and the covariance operator $\mathcal{Q}$. A simplistic special case could be to assume that $\psi(x, y)=\xi(x) \theta(y)$, in which case we find

$$
\sigma(t, s)=\widetilde{\sigma}(s) c \xi(t-s)
$$

for a constant $c$. In this simple example, we can recover interesting LSS processes for the spot price dynamics by choosing $\xi$ appropriately. For example, we can recover so-called continuous-time autoregressive moving average (CARMA) processes by letting

$$
\xi(x)=\mathbf{b}^{*} \exp (A x) \mathbf{e}_{p} .
$$

Here, $\mathbf{e}_{p}$ is the $p$ 'th canonical basis vector in $\mathbb{R}^{p}, p \in \mathbb{N}, \mathbf{b} \in \mathbb{R}^{p}$ is a vector $\mathbf{b}^{*}=$ $\left(b_{0}, b_{1}, \ldots, b_{q}=1,0, \ldots, 0\right)$ for $q<p$, and $A$ is a $p \times p$-matrix of the form

$$
A=\left[\begin{array}{cc}
0 & I \\
-\alpha_{p} & -\alpha_{p-1} \cdots-\alpha_{1}
\end{array}\right]
$$

with $\alpha_{i}>0, i=1, \ldots, p$ and $I$ the $p-1 \times p-1$ identity matrix. We say that we have a volatility modulated $\operatorname{CARMA}(p, q)$ dynamics for the spot for this special choice. Note that we are free to choose the covariance kernel $q$ and the $\theta$, which means that we can have many different forward curve models resulting in the same CARMA spot model. CARMA-processes have been applied to temperature models (see Benth et al. [9] and Härdle and Lopez-Cabrera [30]), spot power prices (see Garcia et al. [28]) and the prices of oil (see Paschke and Prokopczuk [39]). For a detailed analysis of CARMA processes, we refer the reader to Brockwell [16]. 


\subsection{Hilbert-Schmidt Operators}

In this Section, we analyse the Hilbert-Schmidt operators on $H_{w}$. The Hilbert-Schmidt operators on $L^{2}$ are exactly the integral operators where the kernel is an $L^{2}$ function and the Hilbert-Schmidt norm is their $L^{2}$-norm. This can be exploited to identify the Hilbert-Schmidt operators on $H_{w}$ in terms of integral operators, as $H_{w}$ is almost isometric to $L^{2}$.

Lemma 4.9 Let $\mathcal{T}$ be a Hilbert-Schmidt operator on $L^{2}\left(\left(\mathbb{R}_{+}, \mathcal{B}, \lambda\right), \mathbb{R}\right)$. Then there is $b \in L^{2}\left(\left(\mathbb{R}_{+}^{2}, \mathcal{B}_{2}, \lambda_{2}\right), \mathbb{R}\right)$ such that

$$
\mathcal{T} f(x)=\int_{0}^{\infty} b(x, y) f(y) \mathrm{d} y
$$

for any $f \in L^{2}\left(\left(\mathbb{R}_{+}, \mathcal{B}, \lambda\right), \mathbb{R}\right)$ where $\lambda_{2}$ denotes the two-dimensional Lebesgue measure. The Hilbert-Schmidt norm of $\mathcal{T}$ is the $L^{2}$-norm of $b$.

Moreover, if $b$ is an element of $L^{2}\left(\left(\mathbb{R}_{+}^{2}, \mathcal{B}_{2}, \lambda_{2}\right), \mathbb{R}\right)$, then

$$
\mathcal{T} f(x):=\int_{0}^{\infty} b(x, y) f(y) \mathrm{d} y
$$

defines a Hilbert-Schmidt operator.

Proof This is immediate from the isomorphisms from the Hilbert-Schmidt operators to

$$
H:=L^{2}\left(\left(\mathbb{R}_{+}, \mathcal{B}, \lambda\right), \mathbb{R}\right) \otimes L^{2}\left(\left(\mathbb{R}_{+}, \mathcal{B}, \lambda\right), \mathbb{R}\right)
$$

and from $H$ to $L^{2}\left(\left(\mathbb{R}_{+}^{2}, \mathcal{B}_{2}, \lambda_{2}\right), \mathbb{R}\right)$.

The next Lemma shows that the Hilbert-Schmidt operators on a subspace of $H_{w}$ with codimension 1 are integral operators:

Lemma 4.10 Let $\mathcal{C}$ be a closed linear operator on $H_{w}^{0}:=\left\{f \in H_{w}: f(0)=0\right\}$. Then the following statements are equivalent.

(1) $\mathcal{C}$ is a Hilbert-Schmidt operator.

(2) $\mathcal{C}$ is an integral operator and there is $b \in L^{2}\left(\mathbb{R}_{+}^{2}\right)$ such that the kernel of $\mathcal{C}$ is given by

$$
q(x, y):=\int_{0}^{x} \sqrt{w(y) / w(z)} b(z, y) \mathrm{d} z, \quad x, y \in \mathbb{R}_{+} .
$$

If the second statement holds, then the Hilbert-Schmidt norm coincides with the $L^{2}\left(\mathbb{R}_{+}^{2}\right)$-norm of $b$. 
Proof Assume that $\mathcal{C}$ is a Hilbert-Schmidt operator. Then $\mathcal{T}:=\mathcal{W C W}{ }^{-1}$ is a HilbertSchmidt operator where $\mathcal{W}$ is the isomorphism from (15) restricted to $H_{w}^{0}$ and $\mathcal{T}$ and $\mathcal{C}$ have the same Hilbert-Schmidt norm. Lemma 4.9 yields that there is a square integrable function $b$ such that $\mathcal{T} f(x)=\int_{0}^{\infty} b(x, y) f(y) \mathrm{d} y$ and the Hilbert-Schmidt norm coincides with the $L^{2}$-norm of $b$. Since

$$
\mathcal{W}^{-1} g(x)=\int_{0}^{x} \frac{g(y)}{\sqrt{w(y)}} \mathrm{d} y
$$

a short computation yields the representation of $\mathcal{C}$. Indeed, for $f \in H_{w}^{0}$, we have

$$
\begin{aligned}
\mathcal{C} f(x) & =\mathcal{W}^{-1} \mathcal{T} \mathcal{W} f(x) \\
& =\int_{0}^{x} \frac{1}{\sqrt{w(y)}} \mathcal{T} \mathcal{W} f(y) \mathrm{d} y \\
& =\int_{0}^{x} \frac{1}{\sqrt{w(y)}} \int_{0}^{\infty} b(y, z) \mathcal{W} f(z) \mathrm{d} z \mathrm{~d} y \\
& =\int_{0}^{x} \frac{1}{\sqrt{w(y)}} \int_{0}^{\infty} b(y, z) \sqrt{w(z)} f^{\prime}(z) \mathrm{d} z \mathrm{~d} y \\
& =\int_{0}^{\infty} q(x, z) f^{\prime}(z) \mathrm{d} z,
\end{aligned}
$$

where we used Fubini's theorem and $q$ is defined by Eq. (20).

Now assume that $\mathcal{C}$ is an integral operator given by a function as in condition (2). Then $\mathcal{T}$ defined by Eq. (19) is a Hilbert Schmidt-operator. The same equations as before yield that $\mathcal{C}=\mathcal{W}^{-1} \mathcal{T} \mathcal{W}$.

The next Theorem gives a complete characterisation of Hilbert-Schmidt operators on $H_{w}$. The proof makes use of the above Lemma.

Theorem 4.11 Let $\mathcal{C}$ be a Hilbert-Schmidt operator on $H_{w}$. Then there is a squareintegrable function $b: \mathbb{R}_{+}^{2} \rightarrow \mathbb{R}, g, h \in H_{w}$ with $g(0)=0=h(0)$ and $c \in \mathbb{R}$ such that

$$
\mathcal{C} f(x)=c f(0)+\langle g, f\rangle+f(0) h(x)+\int_{0}^{\infty} q(x, z) f^{\prime}(z) \mathrm{d} z
$$

where $q(x, z):=\int_{0}^{x} \sqrt{w(z) / w(y)} b(y, z) \mathrm{d} y$. Moreover, the Hilbert-Schmidt norm of $\mathcal{C}$ is given by 


$$
\|\mathcal{C}\|_{H S}^{2}=c^{2}+\langle g, g\rangle+\langle h, h\rangle+\int_{\mathbb{R}_{+}^{2}} b^{2}(x, y) \mathrm{d} x \mathrm{~d} y .
$$

The dual operator of $\mathcal{C}$ is given by

$$
\mathcal{C}^{*} f(x)=c f(0)+g(x) f(0)+\langle f, h\rangle+\int_{0}^{\infty} q^{*}(x, z) f^{\prime}(z) \mathrm{d} z
$$

where $q^{*}(x, y)=\int_{0}^{x} \sqrt{w(y) / w(z)} b(y, z) \mathrm{d} z$. In particular, $\mathcal{C}$ is symmetric if and only if $b$ is symmetric and $g=h$.

Proof Let $H_{w}^{c}$ be the set of constant functions in $H_{w}$. Then $H_{w}$ is the orthogonal sum of $H_{w}^{c}$ and $H_{w}^{0}$ where $H_{w}^{0}$ is defined in Lemma 4.10. Hence we have

$$
H_{w} \otimes H_{w}=H_{w}^{c} \otimes H_{w}^{c}+H_{w}^{c} \otimes H_{w}^{0}+H_{w}^{0} \otimes H_{w}^{c}+H_{w}^{0} \otimes H_{w}^{0}
$$

where the spaces on the right-hand side are orthogonal to each other. Thus the first claim follows from Lemma 4.10 and the value of the norm from the Pythagorean theorem. The structure of $\mathcal{C}^{*}$ follows from similar arguments.

Theorem 4.11 can be used to find a representation for positive semidefinite traceclass operators. Indeed, any positive semidefinite trace class operator is the square of a symmetric Hilbert-Schmidt operator. Both types of operators play a key role in the stochastic integration theory with values in a Hilbert space, cf. Peszat and Zabczyk [40].

Corollary 4.12 Let $\mathcal{Q}$ be a positive semidefinite trace class operator on $H_{w}$. Then there exist $c \in \mathbb{R}_{+}$and a measurable function $\ell: \mathbb{R}_{+}^{2} \rightarrow \mathbb{R}$ such that $\ell$ is absolutely continuous in its first variable,

(1) $\ell(0, \cdot) / \sqrt{w} \in L^{2}\left(\mathbb{R}_{+}\right)$.

(2) $(x, z) \mapsto \frac{w(z)}{w(x)}\left(\partial_{1} \ell(x, z)\right)^{2}$ is symmetric and integrable.

$$
\begin{aligned}
\mathcal{Q} f(x)= & \left(f(0) c+\int_{0}^{\infty} \ell(0, z) f^{\prime}(z) \mathrm{d} z\right)\left(c+\int_{0}^{x} \ell(0, z) / w(z) \mathrm{d} z\right) \\
& +f(0) \int_{0}^{\infty} \ell(x, z) l(0, z) / w(z) \mathrm{d} z+\int_{0}^{\infty} \int_{0}^{\infty} \ell(x, z) \partial_{1} \ell(z, y) \mathrm{d} z f^{\prime}(y) \mathrm{d} y
\end{aligned}
$$

for any $f \in H_{w}, x \in \mathbb{R}_{+}$. 
(4)

$$
\begin{aligned}
\langle\mathcal{Q} f, f\rangle= & \left(f(0) c+\int_{0}^{\infty} \ell(0, z) f^{\prime}(z) \mathrm{d} z\right)^{2} \\
& +\int_{0}^{\infty}\left(f(0) \ell(0, x)+\int_{0}^{\infty} w(x) \partial_{1} \ell(x, z) f^{\prime}(z) \mathrm{d} y\right)^{2} \frac{1}{w(x)} \mathrm{d} x
\end{aligned}
$$

for any $f \in H_{w}$.

(5) $\operatorname{Tr}(\mathcal{Q})=c^{2}+\int_{0}^{\infty}(q(0, z))^{2} / w(z) \mathrm{d} z+\int_{0}^{\infty}\langle q(x, \cdot), q(x, \cdot)\rangle / w(x) \mathrm{d} x$.

Moreover, if $c \in \mathbb{R}_{+}$and $\ell: \mathbb{R}_{+}^{2} \rightarrow \mathbb{R}$ is measurable, absolutely continuous in its first variable, $\ell$ satisfies (1) and (2) and $\mathcal{Q}$ is defined by (3), then $\mathcal{Q}$ is a positive trace class operator satisfying (4) and (5).

Proof Let $\mathcal{C}$ be a symmetric root of $\mathcal{Q}$. Then $\mathcal{C}$ is a symmetric Hilbert-Schmidt operator on $H_{w}$. Let $b, g, h, c, q$ be as in Theorem 4.11. Since $\mathcal{C}$ is symmetric, we can see from Theorem 4.11 that $g=h$ and $b$ are symmetric. Define $\ell(x, z):=h^{\prime}(z) w(z)+q(x, z)$. Then $\ell(0, \cdot)=w h^{\prime}$ thus $\ell$ satisfies (1). Moreover,

$$
\frac{w(x)}{w(z)}\left(\partial_{1} \ell(x, z)\right)^{2}=b^{2}(x, z), \quad x, z \in \mathbb{R}_{+}
$$

thus $\ell$ satisfies (2). We also have

$$
\begin{aligned}
\mathcal{C} f(x) & =c f(0)+\langle h, f\rangle+f(0) h(x)+\int_{0}^{\infty} q(x, z) f^{\prime}(z) \mathrm{d} z \\
& =f(0)(c+h(x))+\int_{0}^{\infty} \ell(x, z) f^{\prime}(z) \mathrm{d} z
\end{aligned}
$$

and hence

$$
\begin{aligned}
\mathcal{Q} f(x)= & \mathcal{C}^{2} f(x) \\
= & \mathcal{C} f(0)(c+h(x))+\int_{0}^{\infty} \ell(x, z)(\mathcal{C} f)^{\prime}(z) \mathrm{d} z \\
= & \left(f(0) c+\int_{0}^{\infty} \ell(0, z) f^{\prime}(z) \mathrm{d} z\right)(c+h(x)) \\
& +\int_{0}^{\infty} \ell(x, z)\left(f(0) h^{\prime}(z)+\int_{0}^{\infty} \partial_{1} \ell(z, y) f^{\prime}(y) \mathrm{d} y\right) \mathrm{d} z
\end{aligned}
$$




$$
\begin{aligned}
= & \left(f(0) c+\int_{0}^{\infty} \ell(0, z) f^{\prime}(z) \mathrm{d} z\right)\left(c+\int_{0}^{x} \ell(0, z) / w(z) \mathrm{d} z\right) \\
& +f(0) \int_{0}^{\infty} \ell(x, z) l(0, z) / w(z) \mathrm{d} z+\int_{0}^{\infty} \int_{0}^{\infty} \ell(x, z) \partial_{1} \ell(z, y) \mathrm{d} z f^{\prime}(y) \mathrm{d} y
\end{aligned}
$$

for any $f \in H_{w}, x \in \mathbb{R}_{+}$where we used Fubini's theorem for the last equation. Moreover,

$$
\begin{aligned}
\langle\mathcal{Q} f, f\rangle= & \langle\mathcal{C} f, \mathcal{C} f\rangle \\
= & (\mathcal{C} f(0))^{2}+\int_{0}^{\infty}\left((\mathcal{C} f)^{\prime}(x)\right)^{2} w(x) \mathrm{d} x \\
= & \left(f(0) c+\int_{0}^{\infty} \ell(0, z) f^{\prime}(z) \mathrm{d} z\right)^{2} \\
& +\int_{0}^{\infty}\left(f(0) \ell(0, x)+\int_{0}^{\infty} w(x) \partial_{1} \ell(x, z) f^{\prime}(z) \mathrm{d} y\right)^{2} \frac{1}{w(x)} \mathrm{d} x
\end{aligned}
$$

for any $f \in H_{w}, x \in \mathbb{R}_{+}$.

If $\mathcal{T}_{i}$ is an integral operator with kernel $q_{i}, i=1,2,3$ and $\mathcal{T}_{1} \mathcal{T}_{2}=\mathcal{T}_{3}$, then, in many cases, one can represent $q_{3}$ in terms of $q_{1}, q_{2}$. For instance, if we let $\Psi$ in the dynamics of $f$ in (5) have values in the set of integral operators, then the covariance $f$ is given by

$$
\langle\langle f, f\rangle\rangle(t)=\int_{0}^{t} \mathcal{U}_{t-s} \Psi(s) \mathcal{Q} \Psi(s)^{*} \mathcal{U}_{t-s}^{*} \mathrm{~d} s .
$$

$\Psi \mathcal{Q} \Psi^{*}$ is a positive trace class operator and it can be represented by $(\Psi \mathcal{C})(\Psi \mathcal{C})^{*}$ where $\mathcal{C}$ is a symmetric root of $\mathcal{Q}$. Then $\mathcal{C}$ and hence $\Psi \mathcal{C}$ are Hilbert-Schmidt operators and hence they are 'almost' integral operators. If $\Psi$ is chosen to be an integral operator as well, then a formula for the kernel of $\Psi \mathcal{C}$ is obtainable. Let us state general conditions for the connection of such operators.

Theorem 4.13 Let $\mathcal{T}_{i}$ be an integral operator with kernel $q_{i}, i=1,2,3$ and $\mathcal{T}_{3} \subseteq$ $\mathcal{T}_{1} \mathcal{T}_{2}$ where $\mathcal{T}_{3}$ is densely defined. Assume that

(1) $q_{2}$ is absolutely continuous with respect to the first variable,

(2) $\left(z \mapsto \sup \left\{\frac{\left|q_{2}(y, z)\right|}{\sqrt{w(z)}}: y \in\left[y_{0}, y_{0}+1\right]\right\}\right) \in L^{2}\left(\mathbb{R}_{+}\right)$for any $y_{0} \in \mathbb{R}_{+}$,

(3) $\left(z \mapsto \sup \left\{\frac{\left|\partial_{1} q_{2}(y, z)\right|}{\sqrt{w(z)}}: y \in\left[y_{0}, y_{0}+1\right]\right\}\right) \in L^{2}\left(\mathbb{R}_{+}\right)$for any $y_{0} \in \mathbb{R}_{+}$and

(4) $\left.\left(z \mapsto \frac{1}{\sqrt{w(z)}} \int_{0}^{\infty}\left|q_{1}(x, y) \partial_{1} q_{2}(y, z)\right| \mathrm{d} y\right\}\right) \in L^{2}\left(\mathbb{R}_{+}\right)$for any $x \in \mathbb{R}_{+}$. 
Then

$$
q_{3}(x, z)=\int_{0}^{\infty} q_{1}(x, y) \partial_{1} q_{2}(y, z) \mathrm{d} y, \quad x, y \in \mathbb{R}_{+} .
$$

Proof Observe, that Conditions (1) to (3) imply, $z \mapsto \partial_{1} q_{2}(y, z) f^{\prime}(z)$ and $z \mapsto$ $q_{2}(y, z) f^{\prime}(z)$ are integrable for any $y \in \mathbb{R}_{+}$because $\left|f^{\prime}\right| \sqrt{w}$ is square-integrable for any $f \in H_{w}$. Moreover, the uniform condition in $y$ allows to interchange the $z$-integration and the derivative with respect to $y$, hence

$$
\int_{0}^{\infty} \partial_{1} q_{2}(y, z) f^{\prime}(z) \mathrm{d} z=\partial_{y} \int_{0}^{\infty} q_{2}(y, z) f^{\prime}(z) \mathrm{d} z .
$$

Similarily, Condition (4) yields

$$
(y, z) \mapsto q_{1}(x, y) \partial_{1} q_{2}(y, z) f^{\prime}(z)
$$

is integrable for any $f \in H_{w}, x \in \mathbb{R}_{+}$. Thus, we have

$$
\begin{aligned}
\int_{0}^{\infty} q_{3}(x, z) f^{\prime}(z) \mathrm{d} z & =\mathcal{T}_{3} f(x) \\
& =\mathcal{T}_{1}\left(\mathcal{T}_{2} f\right)(x) \\
& =\int_{0}^{\infty} q_{1}(x, y) \partial_{y}\left(\int_{0}^{\infty} q_{2}(y, z) f^{\prime}(z) \mathrm{d} z\right) \mathrm{d} y \\
& =\int_{0}^{\infty} q_{1}(x, y) \int_{0}^{\infty} \partial_{1} q_{2}(y, z) f^{\prime}(z) \mathrm{d} z \mathrm{~d} y \\
& =\int_{0}^{\infty} \int_{0}^{\infty} q_{1}(x, y) \partial_{1} q_{2}(y, z) \mathrm{d} y f^{\prime}(z) \mathrm{d} z
\end{aligned}
$$

for any $f \in \operatorname{dom}\left(\mathcal{T}_{3}\right), x \in \mathbb{R}_{+}$where we used Fubini's theorem in the last step. Since $\operatorname{dom}\left(\mathcal{T}_{3}\right)$ is densely defined a monotone class argument yields

$$
q_{3}(x, z)=\int_{0}^{\infty} q_{1}(x, y) \partial_{1} q_{2}(y, z) \mathrm{d} y,
$$

and the Theorem follows. 
We end this analysis of Hilbert-Schmidt operators with looking at the covariance operator $\mathcal{Q}$, and investigate the correlation implied on the Lévy field $L(t, x)$ driving the noise of the forward dynamics in the case $L$ is $H_{w}$-valued.

Assume $\mathcal{Q}$ is the given as the integral operator

$$
\mathcal{Q} f(x)=\int_{0}^{\infty} q(x, y) f^{\prime}(y) \mathrm{d} y
$$

for some 'nice' kernel function $q$, i.e., the function $h_{x}$ defined in Lemma 3.1 is in the domain of $\mathcal{Q}$. Recall that

$$
\mathrm{E}[L(t, x) L(s, y)]=\mathrm{E}\left[\delta_{x} L(t) \delta_{y} L(s)\right]=t \wedge s\left\langle\mathcal{Q} h_{x}, h_{y}\right\rangle .
$$

We have that

$$
h_{x}^{\prime}(v)= \begin{cases}0, & v>x \\ w^{-1}(v), & v \leq x\end{cases}
$$

Thus,

$$
\mathcal{Q} h_{x}(u)=\int_{0}^{\infty} q(u, v) \mathbf{1}(v \leq x) w^{-1}(v) \mathrm{d} v=\int_{0}^{x} q(u, v) w^{-1}(v) \mathrm{d} v .
$$

Moreover,

$$
\left(\mathcal{Q} h_{x}\right)^{\prime}(u)=\partial_{u} \int_{0}^{x} q(u, v) w^{-1}(v) \mathrm{d} v
$$

But then for $x, y \in \mathbb{R}_{+}^{2}$,

$$
\begin{aligned}
\left\langle\mathcal{Q} h_{x}, h_{y}\right\rangle= & \mathcal{Q} h_{x}(0) h_{y}(0)+\int_{0}^{\infty} w(u)\left(\mathcal{Q} h_{x}\right)^{\prime}(u) h_{y}^{\prime}(u) \mathrm{d} u \\
& =\int_{0}^{x} q(0, v) w^{-1}(v) \mathrm{d} v \\
& +\int_{0}^{\infty} w(u) \partial_{u} \int_{0}^{x} q(u, v) w^{-1}(v) \mathrm{d} v w^{-1}(u) \mathbf{1}(u \leq y) \mathrm{d} u
\end{aligned}
$$




$$
\begin{aligned}
& =\int_{0}^{x} q(0, v) w^{-1}(v) \mathrm{d} v+\int_{0}^{y} \partial_{u} \int_{0}^{x} q(u, v) w^{-1}(v) \mathrm{d} v \mathrm{~d} u \\
& =\int_{0}^{x} q(y, v) w^{-1}(v) \mathrm{d} v .
\end{aligned}
$$

Evidently, the covariance between $L(t, x)$ and $L(s, y)$ will depend on the choice of $q$ as well as the weight function $w$ of the space $H_{w}$. For example, choosing $q(y, v)=$ $\exp (-\delta(|y-v|)$ and $w(v)=\exp (-\alpha v)$ for $\alpha>\delta>0$, then, supposing $y \geq x$ we find that the spatial correlation structure becomes

$$
\operatorname{corr}(L(1, x), L(1, y))=e^{-\frac{1}{2} \delta(y-x)} \sqrt{\frac{1-e^{-(\alpha-\delta) x}}{1-e^{-(\alpha-\delta) y}}} .
$$

Note that this is not stationary in distance $y-x$ between the time to maturities.

Remark 4.14 $\Psi \mathcal{Q} \Psi^{*}$ is a positive trace-class operator and hence it has a unique positive $\operatorname{root} \mathcal{C}$ which is a Hilbert-Schmidt operator. Then Theorem 4.11 yields the existence of a kernel $k: \mathbb{R}_{+} \times \mathbb{R}_{+} \rightarrow \mathbb{R}$ and a function $g: \mathbb{R}_{+} \rightarrow \mathbb{R}$ such that

$$
\mathcal{C} f(x)=g(x) f(0)+\int_{0}^{\infty} k(x, y) f^{\prime}(y) \mathrm{d} y, \quad x \in \mathbb{R}_{+}
$$

for any $f \in H_{w}$. By a similar discussion as above, we can find the covariance (or correlation) structure for the field $f(t, x)$ in terms of $k$. But in the case $\mathcal{Q}$ and $\Psi$ are integral operators with sufficiently regular kernels, we can associate $k$ with these kernels by appealing to Theorem 4.13.

\subsection{Multiplication Operators}

An other useful class of operators are multiplication operators. For example, we recall them appearing in connection with integral operators in Sect. 4.1.

Definition 4.15 Let $f: \mathbb{R}_{+} \rightarrow \mathbb{R}$ be a measurable function and

$$
D_{f}:=\left\{g \in H_{w}: f g \in H_{w}\right\}
$$

The multiplication operator with kernel $f$ is defined by

$$
\mathcal{M}_{f}: \mathcal{D}_{f} \rightarrow H_{w}, g \mapsto f g
$$

We have the following:

Lemma 4.16 The multiplication operator $\mathcal{M}_{f}$ with kernel $f$ is closed. 
Proof Let $\left(g_{n}\right)_{n \in \mathbb{N}}$ be a sequence in $D_{f}$ which converges to some $g$ in $H_{w}$ such that $\mathcal{M}_{f} g_{n}$ converges to some $b$ in $H_{w}$. Let $x \in \mathbb{R}_{+}$. Then we have

$$
\begin{aligned}
b(x) & =\delta_{x}(b) \\
& \leftarrow \delta_{x}\left(\mathcal{M}_{f} g_{n}\right) \\
& =f(x) \delta_{x}\left(g_{n}\right) \\
& \rightarrow f(x) g(x) .
\end{aligned}
$$

Thus $f g=b \in H_{w}$ and hence $g \in D_{f}$ and $\mathcal{M}_{f} g=b$.

As it turns out, under some additional hypothesis on the weight function $w$, the domain $D_{f}$ of $\mathcal{M}_{f}$ is $H_{w}$ if and only if $f \in H_{w}$. In this case, the multiplication operator is continuous. This is the content of the next Theorem:

Theorem 4.17 Assume that $k^{2}:=\int_{0}^{\infty} \frac{1}{w(x)} \mathrm{d} x<\infty$ and let $f: \mathbb{R}_{+} \rightarrow \mathbb{R}$ measurable. Then the following statements are equivalent.

(1) $\mathcal{M}_{f}$ is a continuous linear operator on $H_{w}$,

(2) $\mathcal{M}_{f}$ is everywhere defined and

(3) $f \in H_{w}$.

If $\mathcal{M}_{f}$ is a continuous linear operator, then its operatornorm is atmost $\sqrt{5+4 k^{2}}\|f\|_{w}$ and $\mathcal{M}_{f}^{*} g(x)=\left\langle g, f h_{x}\right\rangle$ where $h_{x}$ is defined as in Lemma 3.1.

Proof The implication (1) $\Rightarrow(2)$ is obvious. For the implication (2) $\Rightarrow(3)$, we observe that $f=\mathcal{M}_{f} 1 \in H_{w}$ where $1 \in H_{w}$ denotes the function which is constant one. The last implication $(3) \Rightarrow(1)$ requires more care:

Let $g \in H_{w}$ and recall from Lemma 3.2 that $\|g\|_{\infty} \leq c\|g\|_{w}$ where $c:=\sqrt{1+k^{2}}$. We have

$$
\begin{aligned}
\|f g\|_{w}^{2}= & f^{2}(0) g^{2}(0)+\int_{0}^{\infty} w(x)\left((f g)^{\prime}(x)\right)^{2} \mathrm{~d} x \\
\leq & \|f\|_{w}^{2}\|g\|_{w}^{2}+\int_{0}^{\infty} w(x)\left(\left(f(x) g^{\prime}(x)\right)^{2}+2 f(x) g(x) f^{\prime}(x) g^{\prime}(x)\right. \\
& \left.+\left(f^{\prime}(x) g(x)\right)^{2}\right) \mathrm{d} x \\
\leq & \|f\|_{w}^{2}\|g\|_{w}^{2}+\|f\|_{\infty}^{2}\|g\|_{w}^{2}+\|f\|_{w}^{2}\|g\|_{\infty}^{2} \\
& +2\|f\|_{\infty}\|g\|_{\infty} \int_{0}^{\infty} w(x)\left|f^{\prime}(x) g^{\prime}(x)\right| \mathrm{d} x \\
\leq & \left(1+2 c^{2}\right)\|f\|_{w}^{2}\|g\|_{w}^{2}+2 c\|f\|_{w}\|g\|_{w}|\langle\tilde{f}, \tilde{g}\rangle| \\
\leq & \left(1+2 c^{2}\right)\|f\|_{w}^{2}\|g\|_{w}^{2}+2 c\|f\|_{w}\|g\|_{w}\|\tilde{f}\|_{w}\|\tilde{g}\|_{w} \\
= & \left(1+4 c^{2}\right)\|f\|_{w}^{2}\|g\|_{w}^{2}
\end{aligned}
$$


where $\tilde{f}(x):=\int_{0}^{x}\left|f^{\prime}(x)\right| \mathrm{d} x, \tilde{g}(x):=\int_{0}^{x}\left|g^{\prime}(x)\right| \mathrm{d} x, x \in \mathbb{R}_{+}$and we used CauchySchwarz inequality for the last inequality. Thus $\mathcal{M}_{f}$ is a continuous linear operator with operator norm at most $\sqrt{1+4 c^{2}}\|f\|_{w}$.

For the representation of the dual operator simply observe that

$$
\mathcal{M}_{f}^{*} g(x)=\left\langle\mathcal{M}_{f}^{*} g, h_{x}\right\rangle=\left\langle g, \mathcal{M}_{f} h_{x}\right\rangle=\left\langle g, f h_{x}\right\rangle
$$

This completes the proof.

As an example, we consider a specific forward dynamics using a multiplication operator to define the diffusion term. Suppose that $w(x)=e^{\alpha x}, x \in \mathbb{R}_{+}$for some $\alpha>0$ for the space $H_{w}$. In this case the assumption $k^{2}=\int_{0}^{\infty} w^{-1}(y) \mathrm{d} y=1 /(2 \alpha)<$ $\infty$ is satisfied. In our example we will assume that the driving noise is a Brownian motion $W$ on the space $H_{w}$, and that $\Psi(t):=\psi(t, f(t)), \psi(t, h):=\mathcal{M}_{h g(t)}$ for any $h \in H_{w}, t \geq 0$ where $g: \mathbb{R}_{+} \rightarrow H_{w}$ is continuous. For simplicity we will assume that $\beta=0$, i.e., no drift in the forward dynamics. Theorem 4.17 yields that $\psi$ satisfies the requirements of Proposition 3.7. In view of Proposition 3.7, there is a stochastic càdlàg process $f$ which is a mild solution to the $H_{w}$-valued stochastic differential equation

$$
\mathrm{d} f(t)=\partial_{x} f(t) \mathrm{d} t+\Psi(f(t)) \mathrm{d} W(t)
$$

with $f(0)=f_{0} \in H_{w}$, i.e.,

$$
f(t)=\mathcal{U}_{t} f_{0}+\int_{0}^{t} \mathcal{U}_{t-s} \psi(t, f(s)) \mathrm{d} W(s), \quad t \in \mathbb{R}_{+} .
$$

Let $B_{T}$ be as in Example 3.10, $G(t, T):=g(t, T-t)$ for $0 \leq t \leq T<\infty$, then we have

$$
F(t, T)=F(0, T)+\int_{0}^{t} F(s, T) G(s, T) \mathrm{d} B_{T}(s),
$$

or

$$
F(t, T)=F(0, T) \mathcal{E}\left(A_{T}(t)\right),
$$

where $\mathcal{E}\left(A_{T}(t)\right)$ is the stochastic exponential of the process

$$
A_{T}(t):=\int_{0}^{t} G(s, T) \mathrm{d} B_{T}(s) .
$$


In particular,

$$
S(t)=f_{0}(t)+\int_{0}^{t} F(s, t) G(s, t) \mathrm{d} B_{t}(s), \quad t \in \mathbb{R}_{+} .
$$

In this example, the dynamics of the forwards are given by a mere stochastic exponential while the spot price process follows a rather complicated dynamic. $A_{T}$ is a Gaussian process with independent increments. For the specific choice $G(t, T)=$ $\exp (-\delta(T-s)) \sigma(T), 0 \leq t \leq T<\infty, \delta>0$, we recover the forward dynamics used in Audet et al. [2]. Here, $\delta$ is used to model the Samuelson effect and the time-inhomogeneous function $\sigma$ covers possible seasonality effects in the diffusion.

We end with a structural result on the space $H_{w}$.

Proposition 4.18 Assume that $k^{2}:=\int_{0}^{\infty} \frac{1}{w(x)} \mathrm{d} x<\infty$. Let $\|f\|:=k_{1}\|f\|_{w}$ for $f \in H_{w}$ where $k_{1}=\sqrt{5+4 k^{2}}$. Then

$$
\left(H_{w},\|\cdot\|\right)
$$

is a separable Banach algebra relative to the pointwise multiplication.

Proof Let $f, g \in H_{w}$. Theorem 4.17 yields

$$
\|f g\|=k_{1}\left\|\mathcal{M}_{f} g\right\|_{w} \leq k_{1}^{2}\|f\|_{w}\|g\|_{w}=\|f\|\|g\| .
$$

The Banach algebraic property of $H_{w}$ turns out to simplify considerably the proof of a technical Lemma stated earlier (see Lemma 3.3). We show the following Corollary on Lipschitz-continuity of the square function:

Corollary 4.19 Assume that $\int_{0}^{\infty} \frac{1}{w(x)} \mathrm{d} x<\infty$. Then

$$
\left\|g_{1}^{2}-g_{2}^{2}\right\|_{w} \leq 3\left\|h_{\infty}\right\|_{w}\left\|g_{1}+g_{2}\right\|_{w}\left\|g_{1}-g_{2}\right\|_{w}
$$

for any $g_{1}, g_{2} \in H_{w}$.

Proof Define $f_{+}:=g_{1}+g_{2}$ and $f_{-}:=g_{1}-g_{2}$. Then Corollary 4.18 yields

$$
\left\|g_{1}^{2}-g_{2}^{2}\right\|_{w}=\left\|f_{+} f_{-}\right\|_{w} \leq \sqrt{1+4 k^{2}}\left\|f_{+}\right\|_{w}\left\|f_{-}\right\|_{w}
$$

Acknowledgments This paper has been developed under financial support of the project 'Managing Weather Risk in Electricity Markets' (MAWREM), funded by the RENERGI-program of the Norwegian Research Council. 


\section{Appendix 1: Some Technical Results}

We present some technical results and considerations which are used in the main text of the paper. Most of these results are known, but collected here for the convenience of the reader.

Riesz Bases

Let $\lambda \in \mathbb{C}, T>0, g_{\lambda}(x):=e^{-\lambda x}$ for any $x \in[0, T)$ and

$$
\mathcal{M}_{\lambda}: L^{2}([0, T), \mathcal{C}) \rightarrow L^{2}([0, T), \mathbb{C}), f \mapsto f g_{\lambda}
$$

Then $\mathcal{M}_{\lambda}$ is an invertible linear operator and

$$
\|f\| \min \left\{1, e^{-T \operatorname{Re}(\lambda)}\right\} \leq\left\|\mathcal{M}_{\lambda} f\right\| \leq\|f\| \max \left\{1, e^{-T \operatorname{Re}(\lambda)}\right\} .
$$

Lemma 4.20 Let $\lambda, T>0$,

$$
\text { cut }: \mathbb{R} \rightarrow[0, T), x \mapsto x-\max \{T z: z \in \mathbb{Z}: T z \leq x\}
$$

and

$$
\begin{aligned}
\mathcal{A}: L^{2}([0, T), \mathbb{C}) & \rightarrow L^{2}\left(\mathbb{R}_{+}, \mathbb{C}\right) \\
f & \mapsto\left(x \mapsto e^{-\lambda x} f(\operatorname{cut}(x))\right) .
\end{aligned}
$$

Then $\mathcal{A}$ is a bounded linear operator and its range is closed in $L^{2}\left(\mathbb{R}_{+}, \mathbb{C}\right)$. Moreover,

$$
\frac{e^{-2 T \lambda}}{1-e^{-2 T \lambda}}\|f\|^{2} \leq\|\mathcal{A} f\|^{2} \leq \frac{1}{1-e^{-2 T \lambda}}\|f\|^{2}
$$

for any $f \in L^{2}([0, T), \mathbb{C})$.

Proof $\mathcal{A}$ is obviously linear. Let $f \in L^{2}([0, T), \mathbb{C})$. Then

$$
\begin{aligned}
\|A f\|^{2} & =\sum_{n=0}^{\infty} \int_{n T}^{n T+T} e^{-2 \lambda x}|f|^{2}(\operatorname{cut}(x)) \mathrm{d} x \\
& =\sum_{n=0}^{\infty} \int_{0}^{T} e^{-2 \lambda x-2 \lambda T n}|f|^{2}(x) \mathrm{d} x \\
& =\sum_{n=0}^{\infty} e^{-2 \lambda T n}\left\|\mathcal{M}_{\lambda} f\right\|^{2}
\end{aligned}
$$


where $\mathcal{M}_{\lambda}$ is defined in (21). The norm estimates on $\mathcal{M}_{\lambda}$ yields

$$
\|\mathcal{A} f\|^{2} \leq \sum_{n=0}^{\infty} e^{-2 \lambda T n}\|f\|^{2}=\frac{1}{1-e^{-2 T \lambda}}\|f\|^{2}
$$

and hence $\mathcal{A}$ is bounded by $\sqrt{\frac{1}{1-e^{-2 T \lambda}}}$. Moreover, the computation at the beginning of this section also implies

$$
\|\mathcal{A} f\|^{2} \geq \sum_{n=0}^{\infty} e^{-2 \lambda T(n+1)}\|f\|^{2}=\frac{e^{-2 \lambda T}}{1-e^{-2 \lambda T}}\|f\|^{2} .
$$

Hence $\mathcal{A}$ is an isomorphism on its range. Consequently, the range of $\mathcal{A}$ is closed.

Definition 4.21 Let $T>0$. The restriction operator is the continuous linear projection given by

$$
\mathcal{R}_{T}: L^{2}\left(\mathbb{R}_{+}, \mathbb{C}\right) \rightarrow L^{2}([0, T), \mathbb{C}),\left.f \mapsto f\right|_{[0, T)}
$$

Lemma 4.22 Let $\lambda, x_{0}>0$, define $g_{n}: \mathbb{R}_{+} \rightarrow \mathbb{C}, x \mapsto \frac{1}{\sqrt{x_{0}}} e^{\left(\frac{2 \pi i n}{x_{0}}-\lambda\right) x}$ and let $V$ be the closed subspace of $L^{2}\left(\mathbb{R}_{+}, \mathbb{C}\right)$ generated by $\left(g_{n}\right)_{n \in \mathbb{Z}}$. Then the following statements hold.

(1) $\left(g_{n}\right)_{n \in \mathbb{Z}}$ is a Riesz basis of $V$ and

(2) The linear operator $\mathcal{B}: V \rightarrow L^{2}\left(\left[0, x_{0}\right], \mathbb{C}\right),\left.f \mapsto f\right|_{\left[0, x_{0}\right]}$ is invertible and continuous.

Proof (1) For any $n \in \mathbb{Z}$ define

$$
e_{n}:\left[0, x_{0}\right] \rightarrow \mathbb{C}, x \mapsto \frac{1}{\sqrt{x_{0}}} e^{\frac{2 \pi i n}{x_{0}} x}
$$

Then $\left(e_{n}\right)_{n \in \mathbb{Z}}$ is an orthonormal basis for $L^{2}\left(\left[0, x_{0}\right], \mathbb{C}\right)$. Let $\mathcal{A}$ be defined as in Lemma 4.20. Then $g_{n}=\mathcal{A} e_{n}$ for any $n \in \mathbb{Z}$. Hence the closed subspace generated by $\left(g_{n}\right)_{n \in \mathbb{Z}}$ is the range of $\mathcal{A}$. The properties of $\mathcal{A}$ which are stated in Lemma 4.20 and Young [46, Theorem 1.9] imply that $\left(g_{n}\right)_{n \in \mathbb{Z}}$ is a Riesz basis of $V$.

(2) Define $\mathcal{B}^{-}:=\mathcal{A} \circ \mathcal{M}_{-\lambda}$ where $\mathcal{M}_{-\lambda}$ is defined in (21). Then $\mathcal{B}$ is continuous, linear and invertible as an operator from $L^{2}\left(\left[0, x_{0}\right], \mathbb{C}\right)$ to $V$. Its inverse $\mathcal{B}$ is also an invertible continuous linear operator and for $n \in \mathbb{N}$ we have $\mathcal{B} g_{n}=\mathcal{M}_{\lambda} e_{n}=$ $\left.g_{n}\right|_{\left[0, x_{0}\right]}$.

Properties of the Stochastic Integral

Proposition 4.23 Let $U$ be a Hilbert space, $T \in \mathbb{R}_{+}, \mathcal{M}_{T}^{2}$ be the set of $U$-valued square integrable martingales with a.s. càdlàg paths defined on the time interval $[0, T]$ 
and $\mathcal{M}_{T, c}^{2}$ be the subset of $\mathcal{M}_{T}^{2}$ which contains all a.s. continuous square integrable martingales. Let

$$
\|\cdot\|_{T}: \mathcal{M}_{T}^{2} \rightarrow \mathbb{R}, M \mapsto\|M\|_{T}:=\sqrt{\mathrm{E}\left(M^{2}(T)\right)} .
$$

Then $\mathcal{M}_{T, c}^{2}$ is a closed subset of the pre-Hilbert space $\left(\mathcal{M}_{T}^{2},\|\cdot\|_{T}\right)$. Moreover, $\|\cdot\|_{T}$ is equivalent to the norm

$$
\|\cdot\|_{T}^{*}: \mathcal{M}_{T}^{2} \rightarrow \mathbb{R}, M \mapsto\|M\|_{T}:=\sqrt{\left.\mathrm{E}\left(\sup \left\{M^{2}(t)\right): t \in[0, T]\right\}\right)}
$$

Let $M, N \in \mathcal{M}_{T}^{2}$. Then $\|M-N\|_{T}=0$ if and only if there is a null set $N \subseteq \Omega$ such that

$$
N(\omega, \cdot)=M(\omega, \cdot)
$$

for all $\omega \in \Omega \backslash N$.

Proof See Prévôt and Röckner [41, Chap. 2].

As a simple consequence of Proposition 4.23 one has the well known

Corollary 4.24 Let $M$ be a square integrable martingale with a.s. càdlàg paths and values in some Hilbert-space $U, H$ be a Hilbert space and $\Psi \in \mathcal{L}_{M}^{2}(H)$. Then

$$
X(t):=\int_{0}^{t} \Psi(s) \mathrm{d} M(s)
$$

defines a square integrable martingale with a.s. càdlàg paths. If M has a.s. continuous paths, then $X$ has a.s. continuous paths.

Proposition 4.25 Let $M$ be a square integrable martingale with a.s. càdlàg paths and values in some Hilbert-space $U, F, H$ be Hilbert spaces, $\Psi \in \mathcal{L}_{M}^{2}(F), I(t):=$ $\int_{0}^{t} \Psi(s) \mathrm{d} M(s), t \in \mathbb{R}_{+}$and $\Gamma \in \mathcal{L}_{I}^{2}(H)$. Then

$$
\Phi(t):=\Gamma(t) \Psi(t), \quad t \in \mathbb{R}_{+}
$$

is an element of $L_{M}^{2}(H)$ and

$$
\int_{0}^{t} \Phi(s) \mathrm{d} M(s)=\int_{0}^{t} \Gamma(s) \mathrm{d} I(s) .
$$

Proof We outline the proof taken from Prévôt and Röckner [41, Chap. 2]: First assume that $\Gamma$ and $\Psi$ are simple. Then $\Phi$ is simple and the equality follows from an elementary computation. General $\Gamma$ and $\Psi$ can be approximated by simple integrands such that $L^{2}(\Omega, H)$-convergence holds. 


\section{References}

1. Andresen, A., Koekebakker, S., Westgaard, S.: Modeling electricity forward prices using the multivariate normal inverse Gaussian distribution. J. Energy Mark. 3(3), 1-23 (2010)

2. Audet, A., Heiskanen, P., Keppo, J., Vehviläinen, A.: Modeling Electricity Forward Curve Dynamics in the Nordic Market. In: Bunn, D.W. (ed.) Modelling Prices in Competitive Electricity Markets, pp. 251-265. Wiley, Chichester (2004)

3. Barndorff-Nielsen, O.E.: Processes of normal inverse Gaussian type. Financ. Stoch. 2(1), 41-68 (1998)

4. Barndorff-Nielsen, O.E., Benth, F.E., Veraart, A.: Modelling energy spot prices by volatility modulated Lévy-driven Volterra processes. Bernoulli 19(3), 803-845 (2013)

5. Barndorff-Nielsen, O.E., Schmiegel, J.: Lévy-based tempo-spatial modelling; with applications to turbulence. Uspekhi Mat. NAUK 59, 65-91 (2004)

6. Barth, A., Benth, F.E.: The forward dynamics in energy markets - infinite dimensional modeling and simulation. To appear in Stochastics (2010)

7. Benth, F.E.: The stochastic volatility model of Barndorff-Nielsen and Shephard in commodity markets. Math. Financ. 21, 595-625 (2011)

8. Benth, F.E., Benth, Š., Koekebakker, S.: Stochastic Modelling of Electricity and Related Markets. World Scientific, Singapore (2008)

9. Benth, F.E., Benth, S.: Modeling and Pricing in Financial Markets for Weather Derivatives. World Scientific, Singapore (2013)

10. Benth, F.E., Kallsen, J., Meyer-Brandis, T.: A non-Gaussian Ornstein-Uhlenbeck process for electricity spot price modeling and derivatives pricing. Appl. Math. Financ. 14, 153-169 (2007)

11. Benth, F.E., Koekebakker, S.: Stochastic modeling of financial electricity contracts. Energy Econ. 30(3), 1116-1157 (2008)

12. Benth, F.E., Krühner, P.: Subordination of Hilbert-space valued Lévy processes. http://arxiv.org/abs/ 1211.6266 (2013)

13. Benth, F.E., Lempa, J.: Optimal portfolios in commodity futures markets. Appear Financ. Stoch 18, 407-430 (2014). doi:10.1007/s00780-013-0224-5

14. Björk, T., Gombani, A.: Minimal realizations of interest rate models. Financ. Stoch. 3, 413-432 (1999)

15. Börger, R., Cartea, A., Kiesel, R., Schindlmayr, G.: Cross-commodity analysis and applications to risk management. J. Futures Mark. 29, 197-217 (2009)

16. Brockwell, P.J.: Continuous-time ARMA process, theory and methods. In: Rao, C.R., Shanbhag, D.N. (eds.) Handbook of Statistics: Stochastic Processes, pp. 249-276. Elsevier, Amsterdam (2001)

17. Bühler, H.: Consistent variance curve models. Financ. Stoch. 10, 178-203 (2006)

18. Carmona, R., Tehranchi, M.: Interest Rate Models: An Infinite Dimensional Stochastic Analysis Perspective. Springer, Berlin Heidelberg New York (2006)

19. Carmona, R., Nadtochiy, S.: Tangent Lévy market models. Financ. Stoch. 16(1), 63-104 (2012)

20. Clewlow, L., Strickland, C.: Energy Derivatives: Pricing and Risk Management. Lacima Publications, London (2000)

21. Delbaen, F., Schachermayer, W.: The fundamental theorem of asset pricing for unbounded stochastic processes. Mathem. Annalen 312, 215-250 (1998)

22. Ekeland, I., Taflin, E.: A theory of bond portfolios. Ann. Appl. Probab. 15, 1260-1305 (2005)

23. Filipović, D., Teichmann, J.: Existence of invariant manifolds for stochastic equations in infinite dimensions. J. Funct. Anal. 197, 398-432 (2003)

24. Filipović, D., Teichmann, J.: Regularity of finite-dimensional realizations for evolution equations. J. Funct. Anal. 197, 433-446 (2003)

25. Filipović, D.: Consistency Problems for Heath-Jarrow-Morton Interest Rate Models, Lecture Notes in Mathematics, vol. 1760. Springer, Berlin (2001)

26. Filipović, D., Teichmann, D., Tappe, S.: Term structure models driven by Wiener process and Poisson measures: existence and positivity. http://arxiv.org/abs/0905.1413 (2009)

27. Frestad, D., Benth, F.E., Koekebakker, S.: Modeling term structure dynamics in the Nordic electricity swap market. Energy J. 21(2), 53-86 (2010)

28. Garcia, I., Klüppelberg, C., Müller, G.: Estimation of stable CARMA models with an application to electricity spot prices. Stat. Mod. 11(5), 447-470 (2010)

29. Grafakos, L.: Classical Fourier Analysis, 2nd edn. Springer, New York (2008)

30. Härdle, W., Lopez Cabrera, B.: The implied market price of weather risk. Appl. Math. Finance 19(1), 59-95 (2012) 
31. Heath, D., Jarrow, R., Morton, A.: Bond pricing and the term structure of interest rates: a new methodology for contingent claims valuation. Econometrica 60, 77-105 (1992)

32. Hull, J.C.: Options, Futures \& Other Derivatives, 4th edn. Prentice Hall, Upper Saddle River (2000)

33. Jacod, J.: Calcul Stochastique et Problèmes de Martingales, Lecture Notes in Mathematics, vol. 714. Springer, Berlin (1979)

34. Jacod, J., Shiryaev, A.: Limit Theorems for Stochastic Processes, 2nd edn. Springer, Berlin (2003)

35. Kallsen, J., Krühner, P.: On a Heath-Jarrow-Morton approach for stock options. To Appear in Finance and Stochastics (2014)

36. Koekebakker, S., Ollmar, F.: Forward curve dynamics in the Nordic electricity market. Manag. Financ 31(6), 74-95 (2005)

37. Lucia, J., Schwartz, E.: Electricity prices and power derivatives: evidence from the Nordic Power Exchange. Rev. Deriv. Res. 5(1), 5-50 (2002)

38. Palmer, T.: Banach Algebras and the General Theory of *-Algebras, vol. I. Cambridge University Press, Cambridge (1994)

39. Paschke, R., Prokopczuk, M.: Commodity derivatives valuation with autoregressive and moving average components in the price dynamics. J. Bank. Financ. 34(11), 2741-2752 (2010)

40. Peszat, S., Zabczyk, J.: Stochastic Partial Differential Equations with Lévy Noise. Cambridge University Press, Cambridge (2007)

41. Prévôt, C., Röckner, M.: A Concise Course on Stochastic Partial Differential Equations. Springer, Berlin (2007)

42. Rydberg, T.H.: The normal inverse Gaussian Lévy process: simulation and approximation. Commun. Stat. -Stoch. Models 13(4), 887-910 (1997)

43. Sato, K.: Lévy Processes and Infinitely Divisible Distributions. Cambridge University Press, Cambridge (1999)

44. Tappe, S.: An alternative approach on the existence of affine realizations for HJM term structure models. Proc. R. Soc. Ser. A 466, 3033-3060 (2010)

45. Tappe, S.: Some refinements of existence results for SPDEs driven by Wiener proceses and Poisson random measure. Intern. J. Stoch. Anal. 18, 24 (2012)

46. Young, R.: An Introduction to Nonharmonic Fourier Series. Academic Press Inc., New York (1980) 